\title{
A systematic review of the effectiveness and cost-effectiveness of peer education and peer support in prisons
}

Anne-Marie Bagnall ${ }^{*}$, Jane South ${ }^{1}$, Claire Hulme², James Woodall1 ${ }^{1}$, Karen Vinall-Collier ${ }^{2}$, Gary Raine ${ }^{1}$, Karina Kinsella', Rachael Dixey ${ }^{1}$, Linda Harris ${ }^{3}$ and Nat MJ Wright ${ }^{4}$

\begin{abstract}
Background: Prisoners experience significantly worse health than the general population. This review examines the effectiveness and cost-effectiveness of peer interventions in prison settings.

Methods: A mixed methods systematic review of effectiveness and cost-effectiveness studies, including qualitative and quantitative synthesis was conducted. In addition to grey literature identified and searches of websites, nineteen electronic databases were searched from 1985 to 2012.

Study selection criteria were:

Population: Prisoners resident in adult prisons and children resident in Young Offender Institutions (YOls).

Intervention: Peer-based interventions.

Comparators: Review questions 3 and 4 compared peer and professionally led approaches.

Outcomes: Prisoner health or determinants of health; organisational/process outcomes; views of prison populations.

Study designs: Quantitative, qualitative and mixed method evaluations.

Results: Fifty-seven studies were included in the effectiveness review and one study in the cost-effectiveness review; most were of poor methodological quality. Evidence suggested that peer education interventions are effective at reducing risky behaviours, and that peer support services are acceptable within the prison environment and have a positive effect on recipients, practically or emotionally. Consistent evidence from many, predominantly qualitative, studies, suggested that being a peer deliverer was associated with positive effects. There was little evidence on cost-effectiveness of peer-based interventions.

Conclusions: There is consistent evidence from a large number of studies that being a peer worker is associated with positive health; peer support services are also an acceptable source of help within the prison environment and can have a positive effect on recipients. Research into cost-effectiveness is sparse.
\end{abstract}

Systematic review registration: PROSPERO ref: CRD42012002349.

Keywords: Systematic review, Prisoners, Prisons, Peer interventions, Peer education, Peer support, Health promotion, Health inequalities

\footnotetext{
* Correspondence: a.bagnall@leedsbeckett.ac.uk

${ }^{1}$ Centre for Health Promotion Research, Leeds Beckett University, Leeds LS1 $3 \mathrm{HE}, \mathrm{UK}$

Full list of author information is available at the end of the article
}

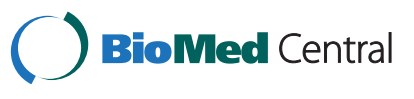

(c) 2015 Bagnall et al.; licensee BioMed Central. This is an Open Access article distributed under the terms of the Creative Commons Attribution License (http://creativecommons.org/licenses/by/4.0), which permits unrestricted use, distribution, and reproduction in any medium, provided the original work is properly credited. The Creative Commons Public Domain Dedication waiver (http://creativecommons.org/publicdomain/zero/1.0/) applies to the data made available in this article, unless otherwise stated. 


\section{Background}

Offender health is a priority for the Department of Health in England and Wales [1] because ill health is more prevalent in prisoners than the general population [2], and prisoners experience significant health inequalities associated with multi-faceted social problems. [3,4] In December 2013, the prison population of England and Wales was 85,429 [5] - high by European standards [6] - with a relative increase in prisoners aged over 50 years [7]. The global prison population has also grown exponentially in all five continents, to a reported 10 million [8]. Imprisonment can produce adverse health impacts, particularly in mental health [9,10]; in 2012, for example, there were 23,158 self-harm incidents in prisons, affecting approximately 6,761 individuals. Younger prisoners, female prisoners and prisoners in the early stages of custody were most likely to self-harm. Suicides are reported to be 102.6 per 100,000 prisoners, compared with $10-12$ per 100,000 in the general population [11]. Evidence shows that prisoners engage in riskier health behaviours, such as drug and alcohol misuse [4] and smoking [12]. Inequalities in long term conditions also exist; with over a quarter of newly sentenced prisoners reporting a long-standing physical disorder or disability [13]. Evidence suggests that women prisoners [13,14] and older prisoners [7] have greater physical health needs.

Since 2006, the NHS has had responsibility for prison healthcare in England and Wales, with a duty to provide services equivalent to those in the community and, since April $1^{\text {st }}$ 2013, NHS England took responsibility and oversight for commissioning all health services (with the exception of some emergency care, ambulance services, out of hours and 111 services) for people in prisons in England through 'Health and Justice' commissioning teams. [15] While many offenders experience barriers accessing health services outside of prison [16], prison health services can potentially improve prisoners' physical and mental health [17]. NHS England have a clear remit for commissioning health promotion in prison, supported by the Ministry of Justice who are responsible for wider health promotion through non-clinical service provision, such as exercise promotion delivered by qualified prison gym staff [15].

Peer-based interventions, where prisoners provide education, support or advice to other prisoners, can contribute to achieving health and social goals within the prison environment and beyond [18]. A 2002 survey estimated that seven percent of prisoners played peer support roles [19]. Justifications include: ability of peers to connect with other prisoners [20] and to have social influence with vulnerable populations resistant to professional advice [21,22]; direct benefits for the peer deliverers themselves [20,23]; wider benefits for the prison system including effective use of resources
$[24,25]$; expanding the range of health services in the criminal justice system [26].

There is evidence of peer interventions operating across prisons globally, ranging from HIV/AIDS programmes in Mozambique and Siberia [27,28] to peer-led emotional support schemes in Israeli prisons [29]. Nevertheless, recent commentators have argued that the emphasis placed on health promotion intervention varies significantly across the world's prison systems. The WHO's healthpromoting prison philosophy, for example, is less well developed in resource-poor regions, like sub-Saharan Africa [30]. This is reiterated in recent reviews which have shown that most published accounts of peer interventions come from prison systems in the UK, US, Canada and Australia [31].

Peer support is an established feature of prison life in England and Wales, for example the Listeners scheme, developed by the Samaritans and first launched in 1991 at HMP Swansea [32] as part of a suicide prevention strategy, now operates across most prisons in England and Wales. Other peer-based interventions in English and Welsh prisons address substance misuse, violence reduction, support for new prisoners, translation services, housing and employment advice and mentoring schemes [23] and, more recently, health trainer schemes [26].

There is an extensive evidence base on peer roles for improving access to healthcare services and removing barriers to health in the general population [33,34], but more needs to be known about the effectiveness of these interventions in prison settings, especially given their prominence $[19,23,35]$. The international literature on effectiveness of different types of prison-based peer education and support has not been systematically reviewed. One literature review of prison-based peer education schemes noted the dearth of evidence demonstrating effectiveness, despite positive impacts reported by some studies [22], while a recent systematic review of peer health promotion concluded that peer education could impact positively on attitudes, knowledge and behaviours of sexual health and HIV prevention, but there was little research on other health issues [31]. Studies of peer support for suicide prevention/self-harm [20,24] report benefits of peer-delivered emotional support, such as decreased prevalence of suicide [36,37]. Peer-based interventions might be more cost-effective than professionally-delivered ones [22]. The cost-effectiveness of peer interventions promoting behavioural change has been assessed in a variety of settings and populations with mixed results [38-40], but to date there has been no systematic review of the cost-effectiveness of peer interventions on health in prison settings. This study thus addresses a knowledge gap by synthesising evidence on a range of peer-based interventions in prison settings and their effectiveness and cost-effectiveness [41]. 


\section{Methods \\ Objectives}

The study used standard systematic review methodology to appraise evidence on effectiveness and cost-effectiveness [42-44] with input from experts in the field, in the form of steering and advisory groups. A full study protocol was developed and peer-reviewed by the study Steering and Advisory Groups prior to publication on PROSPERO (ref: CRD42012002349 http://www.crd.york.ac.uk/prospero/ display_record.asp?ID=CRD42012002349).

The main research question was:

What is the effectiveness and cost effectiveness of peer-based interventions to maintain and improve health in prisons and young offender institutions (YOIs)?

This led to four review questions:

1. What are the effects of peer-based interventions on prisoner health and the determinants of prisoner health?

2. What are the positive and negative impacts of delivering peer-based interventions on health services within prison settings?

3. How do the effects of peer-based approaches compare to those of professionally-led approaches?

4. What is the cost and cost effectiveness of peer-based interventions in prison settings?

This paper reports the findings for review questions 1,3 and 4; review question 2 will be explored in a separate paper.

\section{Data sources}

Sources searched for papers published from 1985 to 2012, with no language restrictions: MEDLINE; PsycINFO; CINAHL; EMBASE: International Bibliography of the Social Sciences (IBSS); ASSIA; Web of Science, Social Science Citation Index; National Criminal Justice Reference Service Abstracts; Social Services Abstracts; Sociological Abstracts; DARE; TRoPHI; DoPHER; Health Evidence Canada; ORB Social Policy Database; Social Care Online; Academic Search Complete; Cochrane and Campbell Collaboration Databases. Electronic contents lists of key journals (Journal of Correctional Health Care, Health Education \& Behavior, Criminal Justice and Behavior) were also searched.

Search terms drew on results from a previous systematic scoping review on lay roles in public health [45], with further search terms identified in consultation with the project steering group.

Additional databases for the cost-effectiveness review were NHS EED and REPEC (IDEAS). Other databases were searched using an adaptation of the economics search filters developed by the NHS Centre for Reviews and Dissemination combined with the search terms used in the effectiveness literature search strategy.
Search strategies are available from the authors on request.

Unpublished (grey) literature was identified from contacts with experts, conference and dissertation abstracts, reference lists of key papers, hand searches of relevant book chapters, and searches of relevant websites. Contacts made with national and international experts included: Offender Health Research Networks (OHRNs); Prison and Offender Research in Social Care and Health (PORSCH); Samaritans (Listeners scheme); Volunteering England; National Offender Management Service (NOMS); PCTs (health trainers); Prison Officers' Association (POA); Action for Prisoners Families; CLINKS; Prison Governors' Association.

Practitioners and academics with expertise were contacted through academic and practice mailing lists.

\section{Study selection}

Two reviewers independently selected studies for inclusion. Any disagreements were resolved by discussion, and a third reviewer if necessary.

\section{Eligibility criteria}

Population: Prisoners resident in prisons and children in YOIs in any country, all ages, male and female.

Intervention: Any peer-based intervention, including peer education, peer support, peer mentoring, befriending, peer counselling and self-help groups, operating within prisons and YOIs in any country. 'Peer' includes prisoners and ex-prisoners delivering interventions to serving prisoners.

Comparators: For Review Questions 3 and 4, studies comparing peer and professionally-led approaches to the same health or social problem. For all other questions, studies with any or no comparator (or usual care).

Outcomes: Studies reporting any effects of peer-based interventions on prisoner health or determinants of health within the prison setting. For review question 4, papers reporting resource use/cost and/or outcome comparisons of peer-based interventions with standard care.

Study designs: Quantitative, qualitative and mixed method evaluations.

\section{Data extraction}

Data were extracted onto piloted electronic forms by one reviewer and checked for accuracy by a second, with reference to a third reviewer if necessary. Data extraction fields included: Bibliographic detail; Population details; Setting/institution details; Intervention details; health or social issue; method of delivery; Outcomes.

Additional data extracted from cost-effectiveness studies were: type of economic evaluation; the basis of costing; source of cost data; cost year and discounting; 
summary of effectiveness and costs; cost-effectiveness/ utility; sensitivity analysis and conclusions as reported by the authors.

Detailed extraction of quantitative data took place into Microsoft Word tables and RevMan 5.0.

Detailed extraction of qualitative data took place into NVivo 9 software, using text conversion of pdf files to import the whole paper. Coding was then applied to methodological and other potential sources of variation (such as population, intervention and settings), as well as results, to allow data to be assembled in the most appropriate way [46-48].

Study authors were contacted for additional or missing information, where needed.

\section{Validity assessment}

Appropriate validity assessment criteria were developed based on published checklists $[44,49]$. Data from grey literature were assessed using the same criteria. Two reviewers assessed each study for validity using piloted forms. Disagreements were resolved by discussion and a third reviewer if necessary. No papers were excluded on the basis of the validity assessment.

Each validity assessment form required the reviewer to make an overall assessment of internal validity and of relevance, based on the completed checklists. These were: $1-3$ for internal validity (where $1=$ good internal validity and $3=$ poor internal validity), and $a-c$ for relevance (where $\mathrm{a}=$ highly relevant and $\mathrm{c}=$ not very relevant).

The quality of cost-effectiveness papers were assessed using a modified version of the Drummond et al. checklist [50]. For papers reporting economic evaluations alongside clinical trials, this was supplemented with reference to the Good Practice Guidance produced by the ISPOR Task Force on Economic Evaluations alongside Clinical Trials [51]. For papers reporting cost-effectiveness models, the checklist was supplemented with reference to the checklist proposed by Drummond et al. [50] and the Good Practice Guidance [51].

\section{Data synthesis}

Quantitative data was synthesised by two reviewers. Where data were suitable for statistical meta-analysis, studies were combined using a fixed effect model to give relative risks with 95\% CIs for binary outcomes and weighted or standardised mean differences with 95\% CIs for continuous outcomes. Statistical heterogeneity was examined using the $\mathrm{X}^{2}$ and $\mathrm{I}^{2}$ statistics, with a $\mathrm{X}^{2} \mathrm{p}$-value of $>0.1$ or an $\mathrm{I}^{2}$ value of $>50 \%$ indicating statistical heterogeneity, in which case, reasons for the heterogeneity would be investigated, and a random effects model would be used.
A thematic synthesis of qualitative studies was undertaken to combine evidence [46] using QSR NVIVO software to manage the data and ensure a transparent process.

A mixed method systematic review design similar to that used by the EPPI-Centre [46] was then used to combine qualitative and quantitative data. For Review Question 1, studies were grouped according to intervention mode and then type of outcome. For Review Questions 1 and 3, qualitative themes on outcomes for peer deliverers and recipients were mapped to quantitative results grouped by intervention mode and then type of outcome [52].

Due to lack of detail given in the included studies, it was not possible to look at the modifying effects of type of institution, prisoner pathway or gender differences.

For the cost-effectiveness review, data were synthesised through a narrative review with tabulation of results of all included studies.

\section{Results}

The effectiveness literature search identified 15,320 potentially relevant papers (Figure 1). 14,963 articles were excluded at the title and abstract screening stage, and 357 articles were obtained and screened in full. 237 papers were subsequently excluded, and we were unable to obtain a further 63 potentially relevant articles, leaving 57 studies included in the review.

Searches for the cost or cost-effectiveness analysis of prison-based peer-interventions identified 1158 titles and abstracts (Figure 2). Twenty six full-text papers were retrieved for assessment. From these, one eligible study was identified, 25 studies were excluded on methodological grounds as none reported costs or cost-effectiveness.

A list of excluded studies is available in the full report [41].

The effectiveness review included 57 studies [19,21,23$29,32,36,37,53-98]$, and one study was included in the review of cost-effectiveness $[99,100]$ (Table 1$)$. Twenty were carried out in the UK (Table 2). Peer education was the most studied intervention mode, followed by peer support (Table 3). Twenty studies looked at HIV/ AIDS/Hepatitis C or other blood borne virus or STI prevention $[21,25,27,28,55,60,63,65,66,68,69,75,78,84,85,87$, $89,93,97,98], 12$ at general health and/ or hygiene,(25, 32, $38,40,119,127,133,136,140,148,149,157,159)$ eight at general emotional support,(146, 151-156, 161) and seven at prevention of suicide or self-harm. [20,24,32,36,56,61,86] (Table 4)

Overall, the internal validity of included studies was quite poor, with only five studies judged to be of good quality [53-57], 18 of moderate quality [23,29,32,58-72] and 32 poor quality $[19,21,25,27,28,36,73-98]$. Five were 


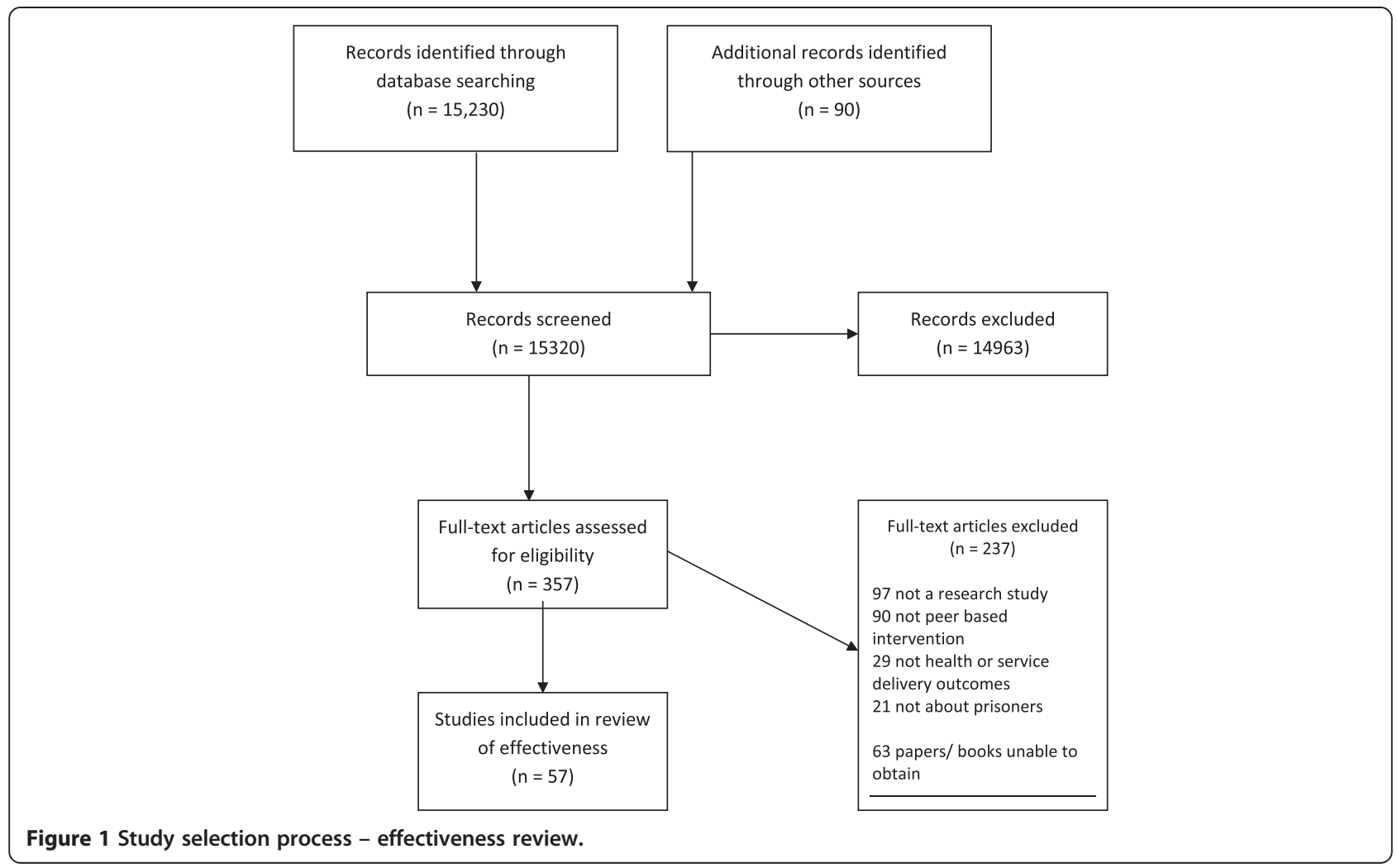

judged to be highly relevant $[53,54,56,57,59]$, with 27 of some relevance $[19,21,23,28,29,32,55,58,60,61,63-68,70$, $71,75,79,81,84,86,92,95-97]$ and 22 not very relevant [25,27,36,62,69,72-74,76-78,80,82,83,85,87-91,93,98].

The main issues affecting internal validity were small sample size, lack of comparators and/or lack of adjustment for potential confounding factors, poor reporting of study methodology and poor reporting of results, limiting meta-analysis of quantitative studies, or metaethnography of qualitative studies. Only two studies defined "peer".

A typology of interventions was developed with working definitions for the major intervention modes (Table 5).

\section{Review Question 1: What are the effects of peer-based interventions on prisoner health?}

Fifty-one studies were relevant to review question 1 [19,21,25,27-29,32,36,53-64,66-77,79-84,86-98], eighteen had a quantitative design [21,25,27-29,60,63,66,69,71, $83,84,86,88,89,92,93,98]$, three of these were RCTs $[25,63,84]$. Fourteen studies had a qualitative design $[32,53,55-57,61,62,67,70,74-77,87,107]$ and 15 were mixed methods [19,36,54,58,59,68,72,79-81,90,94-97]. Four studies had an unclear design $[64,73,82,91]$. Seventeen were UK studies [19,32,53,56,57,59,61,62,67,73,74,88,89,91, $92,94]$ and 17 were from the USA [21,25,55,60,63,64, $66,68,71,77,83,84,86,87,90,93,98]$. The predominant intervention type was peer education (19 studies) [21,25,27-29,55,60,63,66,68,69,76,84,88-90,93,97,98].

Findings are presented in Table 6.

\section{Peer education}

Ten included studies $[28,66,68,69,84,88,90,93,97,98]$ reported the effects of peer education on prisoner knowledge. There was no standard outcome measure used. Statistically significant improvements favouring peer education were seen in the number of correct answers to 22 of the 43 questions asked, while negative effects of peer education were seen in the answers to one of the 43 questions asked. The responses to the remaining 20 questions showed no evidence of effect of the intervention. Risk ratios ranged from 0.43 (95\% CI: 0.33, 0.56, 1 study $\mathrm{n}=949)$ - in favour of peer education to $3.06(95 \%$ CI: $1.91,4.91,1$ study, $\mathrm{n}=200$ ) - against peer education (Figure 3). Qualitative evidence suggested that peer educators improved their own knowledge of health issues as a result of their training $[55,68,76]$.

Findings were equivocal for the effects of peer education on behaviour change intentions and health beliefs. Consistent evidence indicated that peer education reduced risky behaviours: not using a condom at first intercourse after release from prison (pooled RR 0.73, 95\% CI: 0.61, 0.88, 2 studies, $\mathrm{n}=400$ ); injecting drugs after release from prison (pooled RR 0.66, 95\% CI: 0.53, 0.82, 2 studies, $n=400$ ); injected in past 4 weeks (RR 


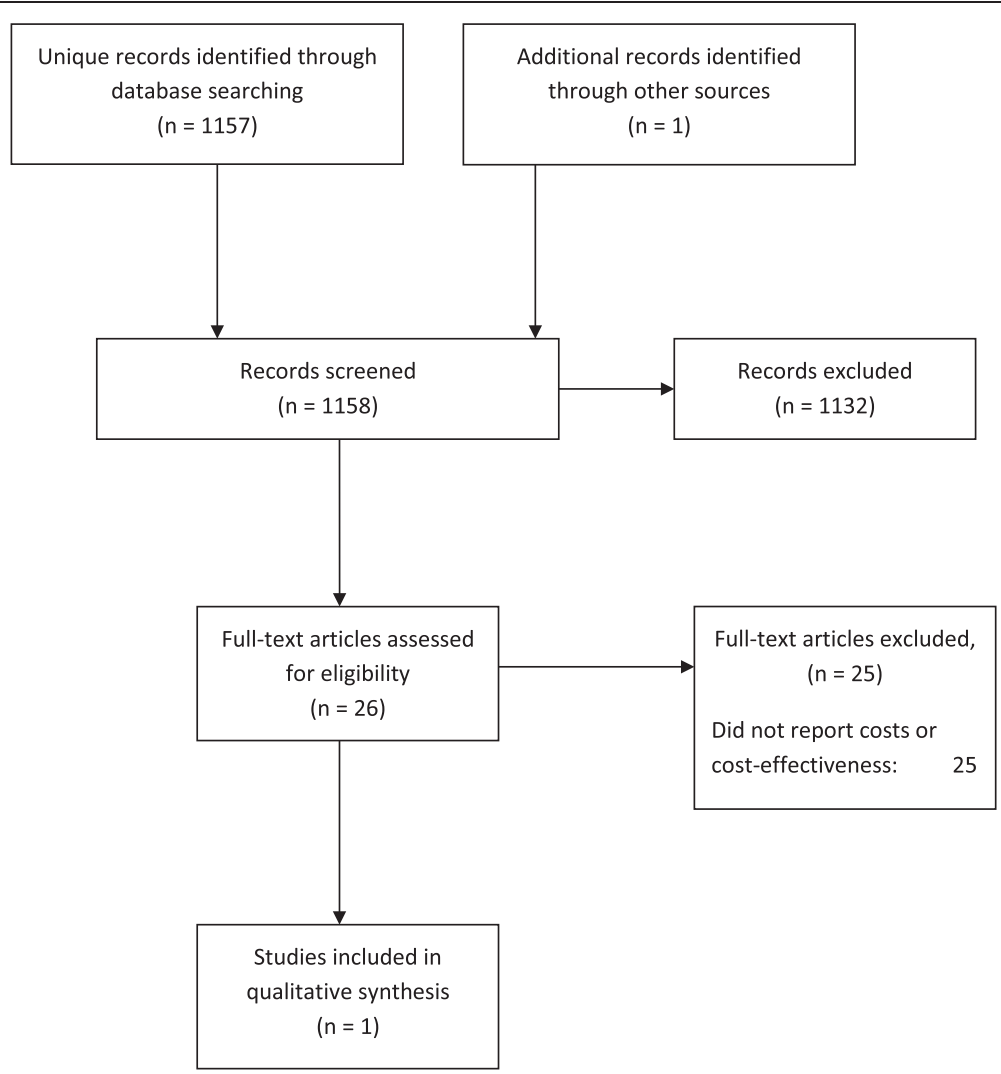

Figure 2 Study selection process - cost-effectiveness review.

0.11, 95\% CI: 0.01, 0.85, 1 study, $\mathrm{n}=241$ ); sharing injection equipment after release from prison (pooled RR 0.33, 95\% CI: 0.20, 0.54, 2 studies, $\mathrm{n}=400$ ); peer educators never having had an HIV test (RR 0.31, 95\% CI: $0.12,0.78,1$ study, $\mathrm{n}=847$ ) (see Figure 2). Weak evidence indicated an association between peer health education programmes and uptake of screening/HIV testing in prisons $[87,89]$.

\section{Peer support}

Six included studies reported the effects of peer support interventions on prisoners [58,79-81,95,96]. These all reported on the Canadian Peer Support Team (PST) program and used similar evaluation designs and outcome measures. The PST Program is a model that has been developed and delivered across a number of Canadian prisons. It is specifically targeted at women prisoners and is based on a holistic, women-centred approach to health care that aims to be culturally sensitive and to develop the women's autonomy and selfesteem. Three studies used the Rosenberg self-esteem scale to measure prisoners' self-esteem $[58,80,95]$ and found no statistically significant effect (pooled WMD 1.51, 95\% CI: $-0.84,3.86,3$ studies, $\mathrm{n}=83$ ), although there was substantial heterogeneity $\left(\mathrm{I}^{2}=81 \%\right)$. Strong qualitative evidence related to improvements in the peer deliverers' self-esteem, self-worth and confidence as a result of the role $[53,58,79-81,96]$. The sense of being trusted by the prison authorities was reported to enable peer deliverers to regain their self-respect $[23,79]$. The notion that peers became more empowered consequentially was alluded to $[58,79,80,95,96]$. Peer support was reported to have helped prisoners either practically, emotionally, or both [58] and could be particularly beneficial for prisoners during the early part of their sentence [62]. In several studies $[23,58,79,80,96]$, peer deliverers gained better selfawareness and perspective on their life as well as developing the skills to deal with their own health and offending issues. One study [23] suggested that the experience of being a peer support worker could reduce the likelihood of re-offending.

The demands placed on peer support workers/counsellors by other prisoners gave individuals a sense of purpose in prison $[23,53,94]$ and this was beneficial for combatting boredom while serving the prison sentence $[23,53]$. However, there were indications that the role could be challenging and onerous and the burden of care of supporting many prisoners could be problematic [53]. 
Table 1 Included Studies

\begin{tabular}{|c|c|c|c|c|c|c|c|c|}
\hline Study & Country & $\begin{array}{l}\text { Study } \\
\text { design }\end{array}$ & $\begin{array}{l}\text { Health } \\
\text { topics }\end{array}$ & $\begin{array}{l}\text { Nature of } \\
\text { intervention/ } \\
\text { scheme }\end{array}$ & Population/setting & Individual outcomes & $\begin{array}{l}\text { Service, delivery or } \\
\text { organisation outcomes }\end{array}$ & $\begin{array}{l}\text { Validity } \\
\text { score* }\end{array}$ \\
\hline \multirow{2}{*}{$\begin{array}{l}\text { Ashton } \\
2010[75]\end{array}$} & \multirow[t]{2}{*}{ Canada } & \multirow[t]{2}{*}{ Qualitative } & \multirow{2}{*}{$\begin{array}{l}\text { HIV/AIDS and } \\
\text { HCV (\& other } \\
\text { infectious } \\
\text { diseases) }\end{array}$} & \multirow[t]{2}{*}{ Peer support } & \multirow{2}{*}{$\begin{array}{l}\text { "Healing Lodge" - a small ( } 28 \text { bed }) \\
\text { minimum/medium security prison for } \\
\text { Aboriginal women, incorporating } \\
\text { Aboriginal healing practices, } \\
\text { meaningfulness and cultural- } \\
\text { connection. Most women are serving } \\
\text { sentences of } 3 \text { years or less. }\end{array}$} & Strengths of programme listed. & \multirow[t]{2}{*}{ Not reported } & \multirow[t]{2}{*}{$3 b$} \\
\hline & & & & & & Staff perceptions. & & \\
\hline $\begin{array}{l}\text { Betts- } \\
\text { Symond } \\
2011[76]\end{array}$ & Ireland & Qualitative & $\begin{array}{l}\text { Health, } \\
\text { hygiene and } \\
\text { cleanliness }\end{array}$ & $\begin{array}{l}\text { Peer } \\
\text { education }\end{array}$ & $\begin{array}{l}700 \text { prisoners in Wheatfield prison, } \\
\text { Dublin Ireland (medium-high security } \\
\text { male prison) and their immediate } \\
\text { family members }\end{array}$ & $\begin{array}{l}\text { Personal development and } \\
\text { changed outlook of the volunteers; } \\
\text { results presented under } 6 \text { themes: } \\
\text { Environment, behaviours, } \\
\text { capabilities, beliefs and values, } \\
\text { identity \& goals. }\end{array}$ & $\begin{array}{l}\text { Relationship between } \\
\text { operational health services } \\
\text { and inmate IRC volunteers. }\end{array}$ & $3 c$ \\
\hline \multirow{4}{*}{$\begin{array}{l}\text { Blanchette } \\
1998[58]\end{array}$} & \multirow[t]{4}{*}{ Canada } & \multirow{4}{*}{$\begin{array}{l}\text { Mixed } \\
\text { Qualitative\& } \\
\text { Quantitative }\end{array}$} & \multirow{4}{*}{$\begin{array}{l}\text { General } \\
\text { emotional/ } \\
\text { mental health, } \\
\text { psychological } \\
\text { support and } \\
\text { counselling }\end{array}$} & \multirow[t]{4}{*}{ Peer support } & \multirow{4}{*}{$\begin{array}{l}\text { Women resident in one of four small } \\
\text { prisons in Canada: Nova Institution; } \\
\text { Etablissement Joliette; Grand Valley } \\
\text { Institution; Edmonton Institution. }\end{array}$} & Self-esteem; & \multirow{4}{*}{$\begin{array}{l}\text { Staff and prisoners' } \\
\text { awareness and perceptions } \\
\text { of the role and functioning of } \\
\text { the PST (surveys); }\end{array}$} & \multirow[t]{4}{*}{$2 b$} \\
\hline & & & & & & $\begin{array}{l}\text { Sociometric tests for understanding } \\
\text { personal and group dynamics; }\end{array}$ & & \\
\hline & & & & & & $\begin{array}{l}\text { Perceptions of the prison } \\
\text { environment (correctional } \\
\text { environment status inventory); }\end{array}$ & & \\
\hline & & & & & & $\begin{array}{l}\text { Staff and prisoners' views, feelings } \\
\text { and ideas about PST (interviews). }\end{array}$ & & \\
\hline \multirow[t]{2}{*}{$\begin{array}{l}\text { Boothby } \\
2011[53]\end{array}$} & \multirow[t]{2}{*}{ UK } & \multirow[t]{2}{*}{ Qualitative } & \multirow{2}{*}{$\begin{array}{l}\text { General } \\
\text { health/ } \\
\text { support }\end{array}$} & \multirow[t]{2}{*}{ Peer support } & Male prison in the UK. & $\begin{array}{l}\text { Insiders perceptions of role and } \\
\text { themselves. }\end{array}$ & \multirow[t]{2}{*}{ Numbers of prison staff } & \multirow[t]{2}{*}{$1 a$} \\
\hline & & & & & $\begin{array}{l}\text { The scheme supports prisoners who } \\
\text { are new to the prison system. }\end{array}$ & prisoners' mood; suicide rates & & \\
\hline \multirow{2}{*}{$\begin{array}{l}\text { Boyce } 2009 \\
{[59]}\end{array}$} & \multirow[t]{2}{*}{ UK } & \multirow[t]{2}{*}{ Mixed } & \multirow{2}{*}{$\begin{array}{l}\text { Housing/ } \\
\text { resettlement }\end{array}$} & \multirow[t]{2}{*}{ Peer advisors } & Serving prisoners in: & skills and self-confidence, work ethic, & Effects on 'professional' time. & \multirow[t]{2}{*}{$2 a$} \\
\hline & & & & & $\begin{array}{l}3 \text { category B prisons (male), } 1 \text { Youth } \\
\text { Offending Institution (male) }\end{array}$ & $\begin{array}{l}\text { sense of control over their lives, work } \\
\text { experience and qualifications. }\end{array}$ & $\begin{array}{l}\text { Staff concerns: potential for } \\
\text { bullying or intimidation and } \\
\text { breaches of confidentiality. }\end{array}$ & \\
\hline \multirow[t]{3}{*}{$\begin{array}{l}\text { Brooker \& } \\
\text { Sirdifield } \\
2007[54]\end{array}$} & \multirow[t]{3}{*}{ UK } & \multirow[t]{3}{*}{$\begin{array}{l}\text { Mixed } \\
\text { Qualitative \& } \\
\text { Quantitative }\end{array}$} & \multirow[t]{3}{*}{$\begin{array}{l}\text { Multiple } \\
\text { health issues }\end{array}$} & \multirow[t]{3}{*}{$\begin{array}{l}\text { Health } \\
\text { Trainers }\end{array}$} & \multirow[t]{3}{*}{$\begin{array}{l}\text { Serving prisoners in } 4 \text { adult prison, } \\
\text { one Young Offenders Institution } \\
\text { and one probation setting }\end{array}$} & $\begin{array}{l}\text { Perceptions of tutors of the Health } \\
\text { Trainers re. confidence; knowledge } \\
\text { of services; communication skills; } \\
\text { ability to assess someone's } \\
\text { readiness to change; self-esteem; } \\
\text { self-worth. }\end{array}$ & $\begin{array}{l}\text { Perceptions of prison-based } \\
\text { trainees re. their role. }\end{array}$ & \multirow[t]{3}{*}{$1 a$} \\
\hline & & & & & & $\begin{array}{l}\text { Perceptions of health trainers re. } \\
\text { knowledge of health issues and } \\
\text { attitude; confidence in sign-posting } \\
\text { individuals to services; changing } \\
\text { own behaviour. }\end{array}$ & $\begin{array}{l}\text { Perceptions of stakeholders } \\
\text { re: }\end{array}$ & \\
\hline & & & & & & & $\begin{array}{l}\text {-workload for prison PE } \\
\text { departments }\end{array}$ & \\
\hline
\end{tabular}




\begin{tabular}{|c|c|c|c|c|c|c|c|c|}
\hline & & & & & & & & \\
\hline $\begin{array}{l}\text { Bryan } \\
2006[60]\end{array}$ & USA & $\begin{array}{l}\text { Quantitative } \\
\text { Pre-test } \\
\text { post-test de- } \\
\text { sign (one } \\
\text { group only). }\end{array}$ & $\begin{array}{l}\text { HIV } \\
\text { prevention }\end{array}$ & $\begin{array}{l}\text { Peer } \\
\text { education }\end{array}$ & $\begin{array}{l}196 \text { serving prisoners in maximum and } \\
\text { minimum security prisons. } 90 \% \text { male, } \\
\text { mean age } 30.4 y \text {. }\end{array}$ & $\begin{array}{l}\text { Knowledge; Perceived risk; Condom } \\
\text { attitudes; Condom norms; Condom } \\
\text { self-efficacy; Condom intentions; } \\
\text { Attitudes for not sharing needles; } \\
\text { Norms for not sharing needles; } \\
\text { Self-efficacy for not sharing } \\
\text { needles; Intentions to not share } \\
\text { needles; Peer education attitudes; } \\
\text { Peer education norms; Peer } \\
\text { education self-efficacy; Peer } \\
\text { education intentions; Peer } \\
\text { education behaviour. }\end{array}$ & Not reported & $2 b$ \\
\hline \multirow[t]{2}{*}{$\begin{array}{l}\text { Chen } \\
2006 \text { [29] }\end{array}$} & \multirow[t]{2}{*}{ Israel } & \multirow[t]{2}{*}{$\begin{array}{l}\text { Quantitative } \\
\text { Pre \& Post }\end{array}$} & \multirow{2}{*}{$\begin{array}{l}\text { General } \\
\text { emotional/ } \\
\text { mental health, } \\
\text { psychological } \\
\text { support and } \\
\text { counselling }\end{array}$} & \multirow[t]{2}{*}{$\begin{array}{l}\text { Peer } \\
\text { counselling }\end{array}$} & $\begin{array}{l}93 \text { male repeat offenders in three } \\
\text { prisons in Israel. (Two maximum } \\
\text { security and one minimum security). }\end{array}$ & Sense of coherence; Meaning in life; & \multirow[t]{2}{*}{ Not reported } & $2 b$ \\
\hline & & & & & Mean age 36 years $(S D=6.35)$. & Anxiety; Depression; Hostility: & & \\
\hline $\begin{array}{l}\text { Cichowlas \& } \\
\text { Chen } 2010 \\
\text { [77] }\end{array}$ & USA & Qualitative & $\begin{array}{l}\text { General } \\
\text { health/ } \\
\text { support }\end{array}$ & $\begin{array}{l}\text { Prison } \\
\text { hospice } \\
\text { volunteers }\end{array}$ & $\begin{array}{l}\text { II/dying prisoners at Dixon Hospice in } \\
\text { Illinois }\end{array}$ & Perceptions of peer deliverers & Not reported & c \\
\hline & \multirow{5}{*}{ USA } & \multirow{5}{*}{$\begin{array}{l}\text { Quantitative } \\
\& \text { Qualitative }\end{array}$} & \multirow{5}{*}{$\begin{array}{l}\text { HIV/AIDS and } \\
\text { HCV (\& other } \\
\text { infectious } \\
\text { diseases) }\end{array}$} & \multirow{5}{*}{$\begin{array}{l}\text { Peer } \\
\text { education }\end{array}$} & \multirow{5}{*}{$\begin{array}{l}\text { All prisoners in USA were covered by } \\
\text { the survey. }\end{array}$} & $\begin{array}{l}\text { 1. Number of HIV positive inmates } \\
\text { in their custody; }\end{array}$ & \multirow{5}{*}{ Not reported } & \\
\hline & & & & & & 2. If they mandated HIV testing; & & \\
\hline & & & & & & $\begin{array}{l}\text { 3. If they provided prison-based } \\
\text { peer programming on HIV. }\end{array}$ & & \\
\hline & & & & & & If answer to Q3 was YES: & & \\
\hline & & & & & & $\begin{array}{l}\text { Extent of HIV peer education, } \\
\text { and other services. }\end{array}$ & & \\
\hline
\end{tabular}

Perceptions of health trainer

clients; issues discussed; services

referred on to.

-training sessions

-Raising risk issues

- engagement with health services

-Change of focus for the gym

-Highlighting a lack of health

services in some areas

-Raising staff awareness of

health issues and/ or services 


\begin{tabular}{|c|c|c|c|c|c|c|c|c|}
\hline \multirow{3}{*}{$\begin{array}{l}\text { Collica } \\
2010[55]\end{array}$} & & & & & & & & \\
\hline & \multirow[t]{2}{*}{ USA } & \multirow[t]{2}{*}{ Qualitative } & \multirow{2}{*}{$\begin{array}{l}\text { HIV/AIDS and } \\
\text { HCV (\& other } \\
\text { infectious } \\
\text { diseases) }\end{array}$} & \multirow[t]{2}{*}{$\begin{array}{l}\text { Peer } \\
\text { education }\end{array}$} & $\begin{array}{l}\text { Aimed at women in prison with HIV/ } \\
\text { AIDS. }\end{array}$ & \multirow[t]{2}{*}{ Role of peers } & \multirow[t]{2}{*}{ Not reported } & \multirow[t]{2}{*}{$1 b$} \\
\hline & & & & & $\begin{array}{l}\text { One maximum and one medium } \\
\text { security prison for women }\end{array}$ & & & \\
\hline \multirow[t]{3}{*}{$\begin{array}{l}\text { Correctional } \\
\text { Service of } \\
\text { Canada } \\
2009[79]\end{array}$} & \multirow[t]{3}{*}{ Canada } & \multirow[t]{3}{*}{$\begin{array}{l}\text { Quantitative } \\
\text { \& Qualitative }\end{array}$} & \multirow[t]{3}{*}{$\begin{array}{l}\text { General } \\
\text { emotional/ } \\
\text { mental health, } \\
\text { psychological } \\
\text { support and } \\
\text { counselling }\end{array}$} & \multirow[t]{3}{*}{ Peer Support } & \multirow[t]{3}{*}{ Women prisoners "in distress" } & $\begin{array}{l}\text { From interviews: predominant } \\
\text { mental health issues of women } \\
\text { prisoners; how these are } \\
\text { addressed in training sessions; } \\
\text { benefits to trained peer } \\
\text { counsellors }\end{array}$ & $\begin{array}{l}\text { Trust between staff and } \\
\text { prisoners }\end{array}$ & \multirow[t]{3}{*}{$\begin{array}{l}3 c \\
\text { quant/ } \\
3 b \text { qual }\end{array}$} \\
\hline & & & & & & $\begin{array}{l}\text { From survey: whether prisoners } \\
\text { value the PST; reasons for asking } \\
\text { to see a peer counsellor; benefits } \\
\text { to service recipients; helpfulness } \\
\text { of peer counsellors; } \\
\text { recommendations for } \\
\text { improvements }\end{array}$ & $\begin{array}{l}\text { Staff becoming part of peer } \\
\text { support team }\end{array}$ & \\
\hline & & & & & & & $\begin{array}{l}\text { Recommendations for } \\
\text { improvements. }\end{array}$ & \\
\hline $\begin{array}{l}\text { Daigle } \\
2007 \text { [24] }\end{array}$ & Canada & $\begin{array}{l}\text { Not } \\
\text { applicable }\end{array}$ & $\begin{array}{l}\text { Suicide/Self } \\
\text { harm }\end{array}$ & Peer support & $\begin{array}{l}\text { Canadian prisons (no further details } \\
\text { reported). }\end{array}$ & Not reported & $\begin{array}{l}\text { Concerns about recruitment, } \\
\text { security and responsibility }\end{array}$ & N/A \\
\hline \multirow{3}{*}{$\begin{array}{l}\text { Davies } \\
1994[32]\end{array}$} & \multirow[t]{3}{*}{ UK } & \multirow[t]{3}{*}{ Qualitative } & \multirow{3}{*}{$\begin{array}{l}\text { Suicide/Self } \\
\text { harm }\end{array}$} & \multirow[t]{3}{*}{ Listeners } & \multirow[t]{3}{*}{ HMP Swansea (adult prison) } & Attempted suicide rate. & staff time. & \multirow[t]{3}{*}{$2 b$} \\
\hline & & & & & & use of the strip cell or care room. & Prison atmosphere. & \\
\hline & & & & & & $\begin{array}{l}\text { Listeners' perceptions (benefits } \\
\text { to Listeners) }\end{array}$ & & \\
\hline \multirow[t]{2}{*}{$\begin{array}{l}\text { Delveaux \& } \\
\text { Blanchette } \\
2000 \text { [80] }\end{array}$} & \multirow[t]{2}{*}{ Canada } & \multirow[t]{2}{*}{$\begin{array}{l}\text { Quantitative } \\
\text { \& Qualitative }\end{array}$} & \multirow[t]{2}{*}{$\begin{array}{l}\text { General } \\
\text { emotional/ } \\
\text { mental health, } \\
\text { psychological } \\
\text { support and } \\
\text { counselling }\end{array}$} & \multirow[t]{2}{*}{ Peer support } & \multirow[t]{2}{*}{$\begin{array}{l}\text { Small women's prison.Women } \\
\text { prisoners, all serving sentences of two } \\
\text { or more years and classified as } \\
\text { minimum or medium security. }\end{array}$} & $\begin{array}{l}\text { Self esteem; Sociometric tests for } \\
\text { understanding personal and } \\
\text { group dynamics; Perceptions of } \\
\text { the prison environment (correctional } \\
\text { environment status inventory) }\end{array}$ & $\begin{array}{l}\text { Staff and prisoners' } \\
\text { awareness and perceptions } \\
\text { of the role and functioning of } \\
\text { the PST (surveys) }\end{array}$ & \multirow[t]{2}{*}{$3 c$} \\
\hline & & & & & & $\begin{array}{l}\text { Staff and prisoners' views, feelings } \\
\text { and ideas about PST (interviews). }\end{array}$ & & \\
\hline $\begin{array}{l}\text { Dhaliwal \& } \\
\text { Harrower } \\
2009[61]\end{array}$ & UK & Qualitative & $\begin{array}{l}\text { Suicide/Self } \\
\text { harm }\end{array}$ & Listeners & $\begin{array}{l}\text { Vulnerable or distressed prisoners, or } \\
\text { those at risk of suicide. }\end{array}$ & $\begin{array}{l}\text { Listeners' own experiences, the } \\
\text { impact on them as individuals, } \\
\text { skills and/or benefits acquired. }\end{array}$ & $\begin{array}{l}\text { Presents findings in relation } \\
\text { to what the prison service } \\
\text { can do to support the } \\
\text { scheme. }\end{array}$ & $2 b$ \\
\hline $\begin{array}{l}\text { Dolan } \\
2004[27]\end{array}$ & Russia & $\begin{array}{l}\text { Quantitative: } \\
\text { pre and post }\end{array}$ & $\begin{array}{l}\text { HIV/AIDS and } \\
\text { HCV (\& other }\end{array}$ & $\begin{array}{l}\text { Peer } \\
\text { education }\end{array}$ & $\begin{array}{l}\text { Male colony for drug-dependent pris- } \\
\text { oners in Siberia. Mean age } 24 \text { (range }\end{array}$ & Whether seen the program booklet? & $\begin{array}{l}\text { Access to bleach and } \\
\text { condoms }\end{array}$ & $3 c$ \\
\hline
\end{tabular}

If answer to Q3 was NO:

How HIV education was

provided and why inmate

prisoners; how these are

From survey: whether prisoners value the PST; reasons for asking

of peer counsellors;

recommendations for

mprovements.

use of the strip cell or care room.

Listeners' perceptions (benefits

Self esteem: Sociometric tests for understanding personal and prisoners, all serving sentences of two or more years and classified as group dynamics; Perceptions of the prison environment (correctional of the role and functioning of mental health, psychologica environment status inventory) 
Table 1 Included Studies (Continued)

\begin{tabular}{|c|c|c|c|c|c|c|c|c|}
\hline & & & $\begin{array}{l}\text { infectious } \\
\text { diseases) }\end{array}$ & & $\begin{array}{l}\text { years served } 1.2 \text { (SD 0.7), } 66 \% \text { impri- } \\
\text { soned for drug related offence. }\end{array}$ & $\begin{array}{l}\text { Whether participated in peer training } \\
\text { education? }\end{array}$ & & \\
\hline & & & & & & $\begin{array}{l}\text { Demographic characteristics; } \\
\text { Knowledge of HIV transmission; } \\
\text { STI and BBVI status; Drug use; } \\
\text { Sexual activity; Tattooing; Access } \\
\text { to bleach and condoms. }\end{array}$ & & \\
\hline \multirow{2}{*}{$\begin{array}{l}\text { Eamon } \\
2012[81]\end{array}$} & \multirow[t]{2}{*}{ Canada } & \multirow{2}{*}{$\begin{array}{l}\text { Quantitative } \\
\& \text { Qualitative }\end{array}$} & \multirow{2}{*}{$\begin{array}{l}\text { General } \\
\text { emotional/ } \\
\text { mental health, } \\
\text { psychological } \\
\text { support and } \\
\text { counselling }\end{array}$} & \multirow[t]{2}{*}{ Peer Support } & \multirow{2}{*}{$\begin{array}{l}\text { Edmonton Institution for Women } \\
\text { population }=65\end{array}$} & Satisfaction with/ performance of PST; & \multirow{2}{*}{$\begin{array}{l}\text { Suggestions for improvement } \\
\text { to number of sessions }\end{array}$} & \multirow[t]{2}{*}{$3 b$} \\
\hline & & & & & & $\begin{array}{l}\text { Hours per week of support provided } \\
\text { by PST members; Time to response } \\
\text { to inmate calls for peer response; } \\
\text { Level of trust in PST members; } \\
\text { Suggestions for improvement; } \\
\text { Improving relationships. }\end{array}$ & & \\
\hline $\begin{array}{l}\text { Edgar } \\
2011[23]\end{array}$ & UK & $\begin{array}{l}\text { Quantitative } \\
\text { \& Qualitative: }\end{array}$ & $\begin{array}{l}\text { Multiple } \\
\text { health issues }\end{array}$ & $\begin{array}{l}\text { Peer support/ } \\
\text { Listeners }\end{array}$ & Not stated & $\begin{array}{l}\text { Various, including Listeners and } \\
\text { other peer roles. }\end{array}$ & $\begin{array}{l}\text { Diverting workload away } \\
\text { from staff. }\end{array}$ & $2 b$ \\
\hline $\begin{array}{l}\text { Farrin } \\
\text { (undated) } \\
\text { [82] }\end{array}$ & Australia & Review & $\begin{array}{l}\text { Multiple } \\
\text { health issues }\end{array}$ & Peer support & At-risk prisoner in 8 state prisons & $\begin{array}{l}\text { Changes in responsibility, } \\
\text { accountability and self-esteem } \\
\text { (Syed \& Blanchette 2000) }\end{array}$ & $\begin{array}{l}\text { Reports the results from } \\
\text { Devilly et al., } 2003 \text { on } \\
\text { changing attitudes and } \\
\text { behaviours; Offender } \\
\text { preference }\end{array}$ & $3 c$ \\
\hline \multirow[t]{2}{*}{$\begin{array}{l}\text { Foster } \\
2011[56]\end{array}$} & \multirow[t]{2}{*}{ UK } & \multirow[t]{2}{*}{ Qualitative } & \multirow[t]{2}{*}{$\begin{array}{l}\text { Suicide/Self } \\
\text { harm }\end{array}$} & \multirow[t]{2}{*}{ Listeners } & \multirow[t]{2}{*}{$\begin{array}{l}\text { Adult category-B local male prison. Op- } \\
\text { erational capacity } 1103\end{array}$} & $\begin{array}{l}\text { Effect on Listeners' personal } \\
\text { development; Self-esteem; } \\
\text { well-being; relationships. }\end{array}$ & \multirow[t]{2}{*}{$\begin{array}{l}\text { Prison environment, burden } \\
\text { on prison staff and health } \\
\text { care professionals. }\end{array}$} & \multirow[t]{2}{*}{$1 \mathrm{a}$} \\
\hline & & & & & & $\begin{array}{l}\text { Numbers of potential suicides and } \\
\text { incidents of self harm. }\end{array}$ & & \\
\hline \multirow[t]{2}{*}{$\begin{array}{l}\text { Goldstein } \\
2009 \text { [83] }\end{array}$} & \multirow[t]{2}{*}{ USA } & \multirow[t]{2}{*}{ Quantitative } & \multirow[t]{2}{*}{$\begin{array}{l}\text { Mental } \\
\text { health/ } \\
\text { Substance } \\
\text { abuse }\end{array}$} & \multirow[t]{2}{*}{$\begin{array}{l}\text { Peer } \\
\text { mentoring }\end{array}$} & $\begin{array}{l}2 \text { correctional facilities. Incarcerated } \\
\text { women with current or history of } \\
\text { behavioural issues and/ or substance } \\
\text { abuse. }\end{array}$ & \multirow{2}{*}{$\begin{array}{l}\text { Adherence to outpatient psychiatric } \\
\text { treatment, including medication } \\
\text { management; Medication } \\
\text { compliance, sobriety \& symptom } \\
\text { reduction; Re-offending; Abstinence } \\
\text { in the use of alcohol or illegal drugs } \\
\text { or misuse of prescription drugs; } \\
\text { Employment or enrolment in an } \\
\text { educational program or completion } \\
\text { of the application process for disability } \\
\text { benefits; Secure treatment, transitional } \\
\text { housing or a permanent place to live. }\end{array}$} & \multirow[t]{2}{*}{ Nor reported } & \multirow[t]{2}{*}{$3 c$} \\
\hline & & & & & $\begin{array}{l}\text { Age range: } 19 \text { to } 59 \mathrm{y} \text { (mean }=35 \mathrm{y} \text { ). } \\
15 \text { out of the } 32 \text { participants had } 5 \text { or } \\
\text { more prior incarcerations. }\end{array}$ & & & \\
\hline \multirow{3}{*}{$\begin{array}{l}\text { Grinstead } \\
1997[84]\end{array}$} & \multirow[t]{3}{*}{ USA } & \multirow{3}{*}{$\begin{array}{l}\text { Quantitative: } \\
\text { RCT }\end{array}$} & \multirow[t]{3}{*}{ HIV } & \multirow{3}{*}{$\begin{array}{l}\text { Peer } \\
\text { education }\end{array}$} & \multirow{3}{*}{$\begin{array}{l}\text { Male inmates at large ( } \mathrm{n}=\text { approx. } \\
5600 \text { ) medium-security state prison. } \\
45 \% \text { had history of injection drug use, } \\
\text { more than } 75 \% \text { of these reported hav- } \\
\text { ing shared equipment. }\end{array}$} & HIV Knowledge; Preference for teacher; & \multirow[t]{3}{*}{ Not reported } & \multirow[t]{3}{*}{$3 b$} \\
\hline & & & & & & $\begin{array}{l}\text { Condom use intention; Bleach use } \\
\text { intention; HIV antibody use intention; }\end{array}$ & & \\
\hline & & & & & & Interested in taking test now. & & \\
\hline $\begin{array}{l}\text { Grinstead } \\
1999[25]\end{array}$ & USA & $\begin{array}{l}\text { Quantitative. } \\
\text { RCT }\end{array}$ & $\begin{array}{l}\text { HIV } \\
\text { prevention }\end{array}$ & $\begin{array}{l}\text { Peer } \\
\text { education }\end{array}$ & $\begin{array}{l}\text { Large state prison for men. Mean age } \\
35 y \text {, spent more than } 9 y \text { of life in } \\
\text { prison. } 90 \% \text { had just completed a }\end{array}$ & Risky behaviour at follow up: & Not reported & $3 c$ \\
\hline
\end{tabular}


Table 1 Included Studies (Continued)

\begin{tabular}{|c|c|c|c|c|c|c|c|c|}
\hline & & & & & $\begin{array}{l}\text { sentence of less than } 5 y \text { and }<10 \% \\
\text { were imprisoned for the first time. }\end{array}$ & $\begin{array}{l}\text { used a condom the first time they } \\
\text { had sex since release; used drugs } \\
\text { since release; injected drugs since } \\
\text { release; shared needles }\end{array}$ & & \\
\hline \multirow[t]{2}{*}{$\begin{array}{l}\text { Hall \& Gabor } \\
2004[36]\end{array}$} & \multirow[t]{2}{*}{ Canada } & \multirow{2}{*}{$\begin{array}{l}\text { Mixed } \\
\text { quantitative } \\
\text { and } \\
\text { qualitative. }\end{array}$} & \multirow[t]{2}{*}{$\begin{array}{l}\text { Suicide } \\
\text { prevention }\end{array}$} & \multirow[t]{2}{*}{ Listeners } & $\begin{array}{l}\text { Medium security prison with capacity } \\
585 \text {. Inmates have committed serious } \\
\text { crimes. }\end{array}$ & \multirow{2}{*}{$\begin{array}{l}\text { personal growth, knowledge of } \\
\text { suicide, self-esteem, communication } \\
\text { skills, and sense of purpose; } \\
\text { support; general program } \\
\text { operation; impact of training; } \\
\text { personal development }\end{array}$} & \multirow[t]{2}{*}{$\begin{array}{l}\text { Findings are reported related } \\
\text { to program implementation }\end{array}$} & \multirow[t]{2}{*}{$3 c$} \\
\hline & & & & & $\begin{array}{l}\text { modal age category } 18-29 y \text {, followed } \\
\text { by } 30-39 y \text {. Length of sentence ranged } \\
\text { from } 2 \text { years to life. }\end{array}$ & & & \\
\hline $\begin{array}{l}\text { Hoover \& } \\
\text { Jurgens } \\
2009[85]\end{array}$ & Moldova & Qualitative & $\begin{array}{l}\text { HIV/AIDS and } \\
\text { HCV (\& other } \\
\text { infectious } \\
\text { diseases) }\end{array}$ & Peer outreach & $\begin{array}{l}7 \text { prisons (6male prisons and } 1 \text { female } \\
\text { prisons) }\end{array}$ & Not reported & Decline in HIV cases & $3 c$ \\
\hline $\begin{array}{l}\text { Hunter \& } \\
\text { Boyce } \\
2009[57]\end{array}$ & UK & Qualitative & $\begin{array}{l}\text { Housing/ } \\
\text { resettlement }\end{array}$ & Peer advisors & $\begin{array}{l}\text { Prisoners requiring housing advice in } 5 \\
\text { prisons in SE England (Three Category } \\
\text { B prisons (male), one young offender } \\
\text { institution (male) and one female open } \\
\text { prison.) }\end{array}$ & $\begin{array}{l}\text { social interaction with others; } \\
\text { experience and qualifications to } \\
\text { assist post-release; self-confidence. }\end{array}$ & $\begin{array}{l}\text { Views of prisoners and staff } \\
\text { re. staff workload and } \\
\text { prisoners' use of their time in } \\
\text { prison. }\end{array}$ & $1 a$ \\
\hline $\begin{array}{l}\text { Jacobson } \\
\text { \& Edgar } \\
\text { (undated) } \\
{[62]}\end{array}$ & UK & Qualitative & $\begin{array}{l}\text { General } \\
\text { health/ } \\
\text { support }\end{array}$ & Peer support & New arrivals at HMP Edinburgh & Effects on prisoners & Use of staff time & $2 c$ \\
\hline $\begin{array}{l}\text { Junker } \\
2005[86]\end{array}$ & USA & Quantitative & $\begin{array}{l}\text { Suicide/Self } \\
\text { harm }\end{array}$ & $\begin{array}{l}\text { Peer } \\
\text { Observers }\end{array}$ & Those prisoners judged to be suicidal & Not reported. & $\begin{array}{l}\text { Number of hours individuals } \\
\text { spent on suicide watch post- } \\
\text { IOP compared to pre-IOP (i.e. } \\
\text { using staff for observations): }\end{array}$ & $3 b$ \\
\hline \multirow{3}{*}{$\begin{array}{l}\text { Levenson } \\
\text { \& Farrant } \\
2002[19]\end{array}$} & \multirow[t]{3}{*}{ UK } & \multirow{3}{*}{$\begin{array}{l}\text { Quantitative } \\
\text { \& Qualitative }\end{array}$} & \multirow{3}{*}{$\begin{array}{l}\text { Multiple } \\
\text { health issues }\end{array}$} & \multirow{3}{*}{$\begin{array}{l}\text { Peer support/ } \\
\text { Listeners. }\end{array}$} & \multirow[t]{3}{*}{ Not stated } & Perceptions of role ( peer supporters) & \multirow[t]{3}{*}{ Not reported } & \multirow{3}{*}{$\begin{array}{l}3 b \\
\text { quant/ } \\
2 b \text { qual }\end{array}$} \\
\hline & & & & & & Self-esteem. & & \\
\hline & & & & & & $\begin{array}{l}\text { finding accommodation and small } \\
\text { amounts of money after release }\end{array}$ & & \\
\hline \multirow[t]{2}{*}{$\begin{array}{l}\text { Martin } 2008 \\
{[63]}\end{array}$} & \multirow[t]{2}{*}{ USA } & \multirow{2}{*}{$\begin{array}{l}\text { Quantitative. } \\
\text { RCT. }\end{array}$} & \multirow[t]{2}{*}{$\begin{array}{l}\text { HIV/ HCV } \\
\text { prevention }\end{array}$} & \multirow[t]{2}{*}{$\begin{array}{l}\text { Peer } \\
\text { education }\end{array}$} & $\begin{array}{l}3 \text { sites: Delaware, Kentucky and } \\
\text { Virginia. }\end{array}$ & \multirow[t]{2}{*}{$\begin{array}{l}\text { The only outcome reported is } \\
\text { condom use during sex. }\end{array}$} & \multirow[t]{2}{*}{ Not reported } & \multirow[t]{2}{*}{$2 b$} \\
\hline & & & & & $N=343$. Mean age $34 y .86 \%$ male. & & & \\
\hline \multirow{2}{*}{$\begin{array}{l}\text { Maull } 1991 \\
\text { [64] }\end{array}$} & \multirow[t]{2}{*}{ USA } & \multirow{2}{*}{$\begin{array}{l}\text { Study design } \\
\text { unclear }\end{array}$} & \multirow{2}{*}{$\begin{array}{l}\text { General } \\
\text { health/ } \\
\text { support }\end{array}$} & \multirow{2}{*}{$\begin{array}{l}\text { Prison } \\
\text { hospice } \\
\text { volunteers }\end{array}$} & \multirow{2}{*}{$\begin{array}{l}\text { III prisoners at U.S. Medical Centre for } \\
\text { Federal Prisoners in Springfield, } \\
\text { Missouri }\end{array}$} & Effects on volunteers; & \multirow{2}{*}{$\begin{array}{l}\text { Retention/attrition of } \\
\text { volunteers }\end{array}$} & \multirow[t]{2}{*}{$2 b$} \\
\hline & & & & & & Effects on prisoners & & \\
\hline $\begin{array}{l}\text { McGowan } \\
2006[87]\end{array}$ & USA & Qualitative & $\begin{array}{l}\text { HIV } \\
\text { counselling }\end{array}$ & $\begin{array}{l}\text { Peer } \\
\text { education }\end{array}$ & $\begin{array}{l}\text { Male prisoners in state prisons in } \\
\text { California, Mississippi, Rhode Island and } \\
\text { Wisconsin. aged between } 18 \text { and } 29 y \text {, } \\
\text { incarcerated for at least } 90 \text { days, } \\
\text { classified as minimum or medium } \\
\text { security level, scheduled for release } \\
\text { within } 14 \text { to } 60 \text { days. }\end{array}$ & $\begin{array}{l}\text { Effect son HIV testing: mandatory } \\
\text { testing at intake, voluntary testing } \\
\text { at medical intake, and voluntary } \\
\text { testing during a peer health } \\
\text { orientation class. }\end{array}$ & Not reported & $3 c$ \\
\hline
\end{tabular}


Table 1 Included Studies (Continued)

\begin{tabular}{|c|c|c|c|c|c|c|c|c|}
\hline $\begin{array}{l}\text { Mentor } 2 \\
\text { work [73] }\end{array}$ & UK & $\begin{array}{l}\text { Study design } \\
\text { unclear }\end{array}$ & Unclear & $\begin{array}{l}\text { Peer } \\
\text { mentoring }\end{array}$ & $\begin{array}{l}\text { Prisoners with mental health problems } \\
\text { at HMP Liverpool. }\end{array}$ & $\begin{array}{l}\text { Self-esteem, confidence and } \\
\text { motivation; Self-worth; } \\
\text { Communication skills, reasoning } \\
\text { and reflection skills; Mental } \\
\text { health and treatment. }\end{array}$ & $\begin{array}{l}\text { Numbers of volunteers and } \\
\text { prisoners being mentored; } \\
\text { effects after release. }\end{array}$ & $3 c$ \\
\hline $\begin{array}{l}\text { Munoz-Plaza } \\
2005[65]\end{array}$ & USA & Qualitative & $\begin{array}{l}\text { HIV/ AIDS and } \\
\text { HCV (\& other } \\
\text { infectious } \\
\text { diseases) }\end{array}$ & $\begin{array}{l}\text { Peer } \\
\text { education }\end{array}$ & $\begin{array}{l}\text { A state correctional facility in California. } \\
\text { Drug treatment program is located on } \\
\text { a medium security prison yard that } \\
\text { houses male inmates. age range } 20-50 \\
\text { years }\end{array}$ & Not reported & Not reported & $2 b$ \\
\hline \multirow{3}{*}{$\begin{array}{l}\text { O'Hagan } \\
2011[88]\end{array}$} & \multirow[t]{3}{*}{ UK } & \multirow[t]{3}{*}{ Quantitative } & \multirow[t]{3}{*}{ Literacy } & \multirow{3}{*}{$\begin{array}{l}\text { Peer } \\
\text { education }\end{array}$} & \multirow[t]{3}{*}{ Serving Young Offenderss at 5 YOls } & Literacy: & \multirow[t]{3}{*}{ Not reported } & \multirow[t]{3}{*}{$3 c$} \\
\hline & & & & & & Impact on learners; & & \\
\hline & & & & & & Impact on mentors & & \\
\hline \multirow[t]{5}{*}{$\begin{array}{l}\text { Peek } 2011 \\
\text { [89] }\end{array}$} & \multirow[t]{5}{*}{ UK } & \multirow[t]{5}{*}{ Quantitative } & \multirow{5}{*}{$\begin{array}{l}\text { Infectious } \\
\text { disease } \\
\text { prevention: } \\
\text { screening and } \\
\text { vaccination. }\end{array}$} & \multirow[t]{5}{*}{$\begin{array}{l}\text { Peer } \\
\text { education }\end{array}$} & \multirow[t]{5}{*}{$\begin{array}{l}\text { Male prisoners at HMP High Down } \\
\text { Category B male local prison. }\end{array}$} & $\begin{array}{l}\text { Hep } B \text { and Hep } C \text { awareness and } \\
\text { vaccination uptake. }\end{array}$ & signposting to healthcare, & \multirow[t]{5}{*}{$3 c$} \\
\hline & & & & & & $\begin{array}{l}\text { Chlamydia awareness and } \\
\text { screening. }\end{array}$ & $\begin{array}{l}\text { Effects on nurses utilising } \\
\text { their time in the prison. }\end{array}$ & \\
\hline & & & & & & & $\begin{array}{l}\text { Effects on barriers between } \\
\text { nursing staff and prisoners. }\end{array}$ & \\
\hline & & & & & & & Prison atmosphere. & \\
\hline & & & & & & & $\begin{array}{l}\text { Changing role/perception of } \\
\text { prisoners. }\end{array}$ & \\
\hline \multirow{4}{*}{$\begin{array}{l}\text { Penn State } \\
\text { Erie } 2001 \\
{[90]}\end{array}$} & \multirow[t]{4}{*}{ USA } & \multirow[t]{4}{*}{$\begin{array}{l}\text { Mixed } \\
\text { methods }\end{array}$} & \multirow[t]{4}{*}{ Parenting } & \multirow[t]{4}{*}{$\begin{array}{l}\text { Peer } \\
\text { education }\end{array}$} & \multirow{4}{*}{$\begin{array}{l}\text { Fathers in prison. State Correctional } \\
\text { Institute at Albion (SCl Albion), in Erie } \\
\text { county. A medium-security institution } \\
\text { for men }\end{array}$} & $\begin{array}{l}\text { contact with children per } \\
\text { month/year; }\end{array}$ & \multirow[t]{4}{*}{$\begin{array}{l}\text { Staff awareness and } \\
\text { perceptions of programme }\end{array}$} & \multirow[t]{4}{*}{$3 c$} \\
\hline & & & & & & $\begin{array}{l}\text { Anger \& Frustration; Knowledge } \\
\text { about their child/children; Parental } \\
\text { Locus of Control; ICAN Fathering } \\
\text { Profile; Total Parenting score }\end{array}$ & & \\
\hline & & & & & & Father's Questionnaire: knowledge, & & \\
\hline & & & & & & attitudes, skills, and behaviors. & & \\
\hline $\begin{array}{l}\text { Player \& } \\
\text { Martin } 1996 \\
\text { [91] }\end{array}$ & UK & $\begin{array}{l}\text { Study design } \\
\text { unclear }\end{array}$ & $\begin{array}{l}\text { Addictions/ } \\
\text { substance } \\
\text { abuse }\end{array}$ & $\begin{array}{l}\text { Peer } \\
\text { counselling }\end{array}$ & $\begin{array}{l}\text { Prisoners with addictions at HMP } \\
\text { Downview }\end{array}$ & drug use; prisoner behaviour & Not reported & $3 c$ \\
\hline \multirow{2}{*}{$\begin{array}{l}\text { Richman } \\
2004 \text { [92] }\end{array}$} & \multirow[t]{2}{*}{ UK } & \multirow[t]{2}{*}{ Quantitative } & \multirow{2}{*}{$\begin{array}{l}\text { General } \\
\text { emotional/ } \\
\text { mental health, } \\
\text { psychological } \\
\text { support and } \\
\text { counselling }\end{array}$} & \multirow[t]{2}{*}{ Listeners } & \multirow[t]{2}{*}{ HMP Manchester } & Change in demeanour. & \multirow{2}{*}{$\begin{array}{l}\text { Effects on staff - peer worker } \\
\text { relationship. }\end{array}$} & \multirow{2}{*}{$3 b$} \\
\hline & & & & & & $\begin{array}{l}\text { Expected effects on release from } \\
\text { prison (on Listeners) }\end{array}$ & & \\
\hline $\begin{array}{l}\text { Ross } 2006 \\
{[66]}\end{array}$ & USA & $\begin{array}{l}\text { Quantitative } \\
\text { Pre \& Post }\end{array}$ & $\begin{array}{l}\text { HIV/ AIDS and } \\
\text { HCV ( \& other } \\
\text { infectious } \\
\text { diseases) }\end{array}$ & $\begin{array}{l}\text { Peer } \\
\text { Education }\end{array}$ & $\begin{array}{l}36 \text { Texas State prison units. Peer } \\
\text { educators and students were } \\
\text { predominantly male, aged } 34-43 \text { y. }\end{array}$ & $\begin{array}{l}\text { HIV-related knowledge; } \\
\text { self-assessed educator skills } \\
\text { among peer educators; }\end{array}$ & $\begin{array}{l}\text { impact of the peer education } \\
\text { program on HIV testing at } \\
\text { participating units }\end{array}$ & $2 b$ \\
\hline
\end{tabular}




\begin{tabular}{|c|c|c|c|c|c|c|c|c|}
\hline $\begin{array}{l}\text { Schinkel \& } \\
\text { Whyte } 2012 \\
\text { [67] }\end{array}$ & UK & Qualitative & $\begin{array}{l}\text { Housing/ } \\
\text { resettlement }\end{array}$ & $\begin{array}{l}\text { Peer } \\
\text { mentoring }\end{array}$ & $\begin{array}{l}\text { Based in Glasgow - prisons not stated. } \\
\text { Prisoners serving sentences of } \\
\text { between three months and four years. } \\
\text { Service offered to eligible prisoners } \\
\text { who are returning to Glasgow, } \\
\text { Renfrewshire and North Lanarkshire. }\end{array}$ & Effects on prisoners & $\begin{array}{l}\text { Staff perceptions of life } \\
\text { coaches' need for support. }\end{array}$ & $2 b$ \\
\hline $\begin{array}{l}\text { Schlapman } \\
\text { \& Cass } \\
2000[93]\end{array}$ & USA & $\begin{array}{l}\text { Quantitative } \\
\text { - pre and } \\
\text { post }\end{array}$ & $\begin{array}{l}\text { HIV } \\
\text { prevention }\end{array}$ & $\begin{array}{l}\text { Peer } \\
\text { education }\end{array}$ & $\begin{array}{l}\text { Incarcerated adolescents in North } \\
\text { central Indiana juvenile facility. }\end{array}$ & $\begin{array}{l}\text { AIDS knowledge \& self reported } \\
\text { sexual behaviours. }\end{array}$ & Not reported & $3 c$ \\
\hline $\begin{array}{l}\text { Scott } 2004 \\
{[68]}\end{array}$ & USA & $\begin{array}{l}\text { Mixed } \\
\text { quantitative } \\
\text { (pre and } \\
\text { post) and } \\
\text { qualitative) }\end{array}$ & $\begin{array}{l}\text { HIV } \\
\text { prevention }\end{array}$ & $\begin{array}{l}\text { Peer } \\
\text { education }\end{array}$ & $\begin{array}{l}\text { Prisoners at } 5 \text { Texas prison facilities. A } \\
\text { diversity of facilities was selected (small } \\
\text { and large, short and long term, male } \\
\text { and female prisoners) }\end{array}$ & $\begin{array}{l}\text { HIV related knowledge, attitudes } \\
\text { and beliefs among peer educators } \\
\text { and students. }\end{array}$ & $\begin{array}{l}\text { Factors affecting } \\
\text { implementation, } \\
\text { maintenance and overall } \\
\text { impact of the program from } \\
\text { the perspective of program } \\
\text { coordinators, wardens and } \\
\text { peer educators. }\end{array}$ & $\begin{array}{l}2 \mathrm{~b} \\
\text { quant/ } \\
2 \mathrm{c} \text { qual }\end{array}$ \\
\hline \multirow[t]{2}{*}{$\begin{array}{l}\text { Sifunda } \\
2008 \\
{[69,101]}\end{array}$} & \multirow[t]{2}{*}{ South Africa } & \multirow[t]{2}{*}{$\begin{array}{l}\text { Quantitative } \\
\text { Pre \& Post }\end{array}$} & \multirow[t]{2}{*}{$\begin{array}{l}\text { HIV/ AIDS and } \\
\text { HCV ( \& other } \\
\text { infectious } \\
\text { diseases) }\end{array}$} & \multirow[t]{2}{*}{$\begin{array}{l}\text { Peer } \\
\text { education }\end{array}$} & \multirow{2}{*}{$\begin{array}{l}4 \text { medium-sized correctional facilities } \\
\text { (male) in South Africa. Number housed } \\
\text { comparable in size to UK prison..N = } \\
\text { 263. Mean age } 27 \text { y (range 17-55). } \\
\text { Mean period of incarceration = } 2 \text { years } \\
\text { (range } 6 \mathrm{~m}-17 \text { y).65\% were first time } \\
\text { offenders. }\end{array}$} & $\begin{array}{l}\text { Knowledge and beliefs; Attitudes; } \\
\text { Sexual communication, social } \\
\text { norms about gender relations } \\
\text { and sexual violence; }\end{array}$ & \multirow[t]{2}{*}{ Not reported } & \multirow[t]{2}{*}{$2 c$} \\
\hline & & & & & & Self-efficacy; Intentions & & \\
\hline \multirow[t]{2}{*}{$\begin{array}{l}\text { Sirdifield } \\
2006[70]\end{array}$} & \multirow[t]{2}{*}{ UK } & \multirow[t]{2}{*}{ Qualitative } & \multirow{2}{*}{$\begin{array}{l}\text { General } \\
\text { health/ } \\
\text { support }\end{array}$} & \multirow[t]{2}{*}{ Health Trainer } & \multirow[t]{2}{*}{ All prisoners } & $\begin{array}{l}\text { Changes in Health Trainers' attitudes } \\
\text { and health behaviour. }\end{array}$ & \multirow{2}{*}{$\begin{array}{l}\text { demands placed on prison } \\
\text { staff and health services as a } \\
\text { result of the intervention. }\end{array}$} & \multirow[t]{2}{*}{$2 b$} \\
\hline & & & & & & Recognising stress in other prisoners. & & \\
\hline \multirow[t]{6}{*}{$\begin{array}{l}\text { Snow } 2002 \\
{[37]}\end{array}$} & \multirow[t]{6}{*}{ UK } & \multirow[t]{6}{*}{ Quantitative } & \multirow[t]{6}{*}{$\begin{array}{l}\text { Suicide/ self } \\
\text { harm }\end{array}$} & \multirow[t]{6}{*}{ Listeners } & \multirow{6}{*}{$\begin{array}{l}5 \text { prisons having a Samaritan } \\
\text { supported Listener scheme. All prisons } \\
\text { were local type establishments and } \\
\text { chosen because of the comparatively } \\
\text { high rate of suicide. }\end{array}$} & $\begin{array}{l}\text { Perceived benefit from using the } \\
\text { scheme: }\end{array}$ & \multirow[t]{6}{*}{ Not reported } & \multirow[t]{6}{*}{$2 b$} \\
\hline & & & & & & Approachability of listeners & & \\
\hline & & & & & & Availability of listeners & & \\
\hline & & & & & & Use of listener scheme in the future. & & \\
\hline & & & & & & Reasons for not using the scheme & & \\
\hline & & & & & & Ways to improve the scheme & & \\
\hline \multirow[t]{2}{*}{$\begin{array}{l}\text { Stewart } \\
2011[94]\end{array}$} & \multirow[t]{2}{*}{ UK } & \multirow[t]{2}{*}{$\begin{array}{l}\text { Quantitative } \\
\text { \& Qualitative }\end{array}$} & \multirow[t]{2}{*}{$\begin{array}{l}\text { General } \\
\text { health/ } \\
\text { support }\end{array}$} & \multirow[t]{2}{*}{ Peer support } & 3 UK prisons. & Effects on prisoner-carers & $\begin{array}{l}\text { communication between } \\
\text { staff and prisoners. Training } \\
\text { and supervision issues. }\end{array}$ & \multirow[t]{2}{*}{$3 c$} \\
\hline & & & & & $\begin{array}{l}\text { Originally for older prisoners but to } \\
\text { include those with learning disabilities, } \\
\text { mental health problems and prisoners }\end{array}$ & & $\begin{array}{l}\text { Contribution to the health } \\
\text { and social care services } \\
\text { within the gaol. }\end{array}$ & \\
\hline
\end{tabular}

Diffusion of HIV-related

knowledge;

HIV-testing behavior and intentions 
Table 1 Included Studies (Continued)

\begin{tabular}{|c|c|c|c|c|c|c|c|c|}
\hline \multirow[t]{2}{*}{$\begin{array}{l}\text { Syed \& } \\
\text { Blanchette } \\
2000 \text { [95] }\end{array}$} & \multirow[t]{2}{*}{ Canada } & \multirow[t]{2}{*}{$\begin{array}{l}\text { Quantitative } \\
\& \text { Qualitative }\end{array}$} & \multirow[t]{2}{*}{$\begin{array}{l}\text { General } \\
\text { emotional/ } \\
\text { mental health, } \\
\text { psychological } \\
\text { support and } \\
\text { counselling }\end{array}$} & \multirow[t]{2}{*}{ Peer Support } & $\begin{array}{l}\text { Small women's prison, } n=78 \text { at time } \\
\text { of study. All were serving sentences of } \\
\text { minimum } 2 \text { years and were rated at } \\
\text { 'minimum' or 'medium' security levels. }\end{array}$ & $\begin{array}{l}\text { Self esteem; Sociometric tests for } \\
\text { understanding personal and group } \\
\text { dynamics; Perceptions of the prison } \\
\text { environment (correctional environment } \\
\text { status inventory); }\end{array}$ & \multirow[t]{2}{*}{$\begin{array}{l}\text { Staff and prisoners' } \\
\text { awareness and perceptions } \\
\text { of the role and functioning of } \\
\text { the PST (surveys); }\end{array}$} & \multirow[t]{2}{*}{$\begin{array}{l}\text { 3b } \\
\text { quant/ } \\
\text { 1c qual }\end{array}$} \\
\hline & & & & & $\begin{array}{l}\text { Survey respondents, average age } 34.5 \mathrm{y} \\
(\mathrm{sd}=9.07 \text {, range } 21-58) \text {. Average } \\
\text { sentence length } 4.39 \mathrm{y} \text { (range } 2 \text { to } 15 \mathrm{y}) . \\
\text { Average time spent at Grand Valley }= \\
9 \text { months ( } \mathrm{SD}=0.62, \text { range }=2 \text { weeks } \\
\text { to } 2 \text { years). }\end{array}$ & $\begin{array}{l}\text { Staff and prisoners' views, feelings } \\
\text { and ideas about PST (interviews). }\end{array}$ & & \\
\hline \multirow[t]{2}{*}{$\begin{array}{l}\text { Syed \& } \\
\text { Blanchette } \\
2000 \text { [96] }\end{array}$} & \multirow[t]{2}{*}{ Canada } & \multirow[t]{2}{*}{$\begin{array}{l}\text { Quantitative } \\
\& \text { Qualitative }\end{array}$} & \multirow[t]{2}{*}{$\begin{array}{l}\text { General } \\
\text { emotional/ } \\
\text { mental health, } \\
\text { psychological } \\
\text { support and } \\
\text { counselling }\end{array}$} & \multirow[t]{2}{*}{ Peer Support } & $\begin{array}{l}\text { women's prison in Canada. } \mathrm{N}=56 \text { at } \\
\text { time of study. All were serving } \\
\text { sentences of minimum } 2 \text { years and } \\
\text { were rated at 'minimum' or 'medium' } \\
\text { security levels. }\end{array}$ & $\begin{array}{l}\text { Self esteem; Sociometric tests for } \\
\text { understanding personal and group } \\
\text { dynamics; Perceptions of the prison } \\
\text { environment (correctional environment } \\
\text { status inventory); }\end{array}$ & \multirow[t]{2}{*}{$\begin{array}{l}\text { Staff and prisoners' } \\
\text { awareness and perceptions } \\
\text { of the role and functioning of } \\
\text { the PST (surveys) }\end{array}$} & \multirow[t]{2}{*}{$\begin{array}{l}3 \mathrm{~b} \\
\text { quant/ } \\
2 \mathrm{~b} \text { qual }\end{array}$} \\
\hline & & & & & $\begin{array}{l}\text { All women, average age } 35.1 \mathrm{y}(\mathrm{SD}= \\
11.3 \text {, range }=21 \text { to } 62 \text { ). Average } \\
\text { sentence length } 4.7 \text { years (range } 2 \mathrm{y} \text { to } \\
\text { life). Mean time served at Joliette = } \\
13.3 \mathrm{~m} \text { (range } 2 \mathrm{~m} \text { to } 2.5 \mathrm{y} \text { ). }\end{array}$ & $\begin{array}{l}\text { Staff and prisoners' views, feelings and } \\
\text { ideas about PST (interviews). }\end{array}$ & & \\
\hline $\begin{array}{l}\text { Taylor } \\
1994[97]\end{array}$ & Australia & $\begin{array}{l}\text { Quantitative } \\
\text { and } \\
\text { Qualitative: } \\
\text { Pre-post }\end{array}$ & $\begin{array}{l}\text { HIV } \\
\text { prevention }\end{array}$ & $\begin{array}{l}\text { Peer } \\
\text { education }\end{array}$ & $\begin{array}{l}\text { New South Wales Correctional Centres. } \\
90 \% \text { of inmates had been in other } \\
\text { correctional centres. }\end{array}$ & Knowledge; attitudes & $\begin{array}{l}\text { Awareness of the peer } \\
\text { education scheme. }\end{array}$ & $3 b$ \\
\hline $\begin{array}{l}\text { The } \\
\text { Learning } \\
\text { Ladder Ltd. } \\
\text { (undated) } \\
\text { [74] }\end{array}$ & UK & Qualitative. & $\begin{array}{l}\text { Mentoring for } \\
\text { education/to } \\
\text { improve } \\
\text { qualifications }\end{array}$ & $\begin{array}{l}\text { Peer } \\
\text { mentoring }\end{array}$ & $\begin{array}{l}\text { HM Young Offenders Institution } \\
\text { Reading - a small prison holding } \\
\text { prisoners between the ages of } 18 \text { and } \\
21 y \text {. }\end{array}$ & $\begin{array}{l}\text { self-esteem; confidence; attitude to } \\
\text { offending behaviour. }\end{array}$ & Success of scheme. & $3 c$ \\
\hline $\begin{array}{l}\text { Vaz } 1996 \\
{[28]}\end{array}$ & Mozambique & $\begin{array}{l}\text { Quantitative, } \\
\text { pre-post }\end{array}$ & $\begin{array}{l}\text { HIV/ STD } \\
\text { prevention }\end{array}$ & $\begin{array}{l}\text { Peer } \\
\text { education }\end{array}$ & $\begin{array}{l}\text { Largest prison in Mozambique ( } 1900 \\
\text { prisoners incarcerated at time of } \\
\text { study). } 300 \text { inmates sentenced to } \\
1 \text { year or longer, selected on entry. } \\
\text { Mean age } 26 y \text {. }\end{array}$ & $\begin{array}{l}\text { knowledge around HIV/AIDS; } \\
\text { relationship between knowledge } \\
\text { of HIV/AIDS and educational } \\
\text { attainment of participants. }\end{array}$ & Not reported & $3 b$ \\
\hline \multirow[t]{2}{*}{$\begin{array}{l}\text { Walrath } \\
2001[71]\end{array}$} & \multirow[t]{2}{*}{ USA } & \multirow[t]{2}{*}{$\begin{array}{l}\text { Quantitative } \\
\text { Pre \& Post }\end{array}$} & \multirow[t]{2}{*}{ Violence } & \multirow[t]{2}{*}{ Peer training. } & $\begin{array}{l}\text { Medium all-male security corrections } \\
\text { facility in Maryland, USA, housing in- } \\
\text { mates serving sentences of } 3 \text { months } \\
\text { or longer. }\end{array}$ & \multirow[t]{2}{*}{$\begin{array}{l}\text { Anger; Self esteem; Optimism; } \\
\text { Locus of Control; Behaviour }\end{array}$} & \multirow[t]{2}{*}{ Not reported } & \multirow[t]{2}{*}{$2 b$} \\
\hline & & & & & $\begin{array}{l}\text { Age range: } 18 \text { to } 51 \mathrm{y} \text {, mean age } 30 \mathrm{y} \text {. } \\
\text { Average sentence } 20 \mathrm{y} \text {, ranging from } \\
\text { less than } 1 \text { year to life. }\end{array}$ & & & \\
\hline $\begin{array}{l}\text { Wright \& } \\
\text { Bronstein } \\
2007 \\
{[72,102] 2} \\
\text { papers }\end{array}$ & USA & $\begin{array}{l}\text { Mixed } \\
\text { Qualitative \& } \\
\text { Quantitative }\end{array}$ & $\begin{array}{l}\text { General } \\
\text { health/ } \\
\text { support }\end{array}$ & $\begin{array}{l}\text { Prison } \\
\text { hospice } \\
\text { volunteers }\end{array}$ & $\begin{array}{l}\text { Dying prisoners in } 14 \text { prison hospices } \\
\text { in the USA }\end{array}$ & Not reported & $\begin{array}{l}\text { Impact of having a hospice } \\
\text { (\& implicitly, using prisoner } \\
\text { volunteers) on prison } \\
\text { environment \& climate. }\end{array}$ & $2 c$ \\
\hline
\end{tabular}


Table 1 Included Studies (Continued)

\begin{tabular}{|c|c|c|c|c|c|c|c|c|}
\hline \multirow{2}{*}{$\begin{array}{l}\text { Zack } 2001 \\
{[21]}\end{array}$} & \multirow[t]{2}{*}{ USA } & Quantitative & \multirow{2}{*}{$\begin{array}{l}\text { HIV/AIDS and } \\
\text { HCV (\& other } \\
\text { infectious } \\
\text { diseases) }\end{array}$} & \multirow{2}{*}{$\begin{array}{l}\text { Peer } \\
\text { education }\end{array}$} & \multirow{2}{*}{$\begin{array}{l}\text { Medium-security prison housing } \\
\text { approximately } 6000 \text { men who stay at } \\
\text { the prison for an average of less than } \\
\text { two years. Men arriving at and leaving } \\
\text { the prison, and women visitors. }\end{array}$} & \multirow{2}{*}{$\begin{array}{l}\text { Intentions to use condoms and } \\
\text { be tested for HIV; Knowledge; } \\
\text { HIV/AIDS testing; behaviour }\end{array}$} & Resistance from staff & \multirow[t]{2}{*}{$3 b$} \\
\hline & & $\mathrm{RCT}$ & & & & & Institutional lockdowns & \\
\hline $\begin{array}{l}\text { Zucker } 2006 \\
\text { [98] }\end{array}$ & USA & $\begin{array}{l}\text { Quantitative. } \\
\text { One-group } \\
\text { pretest - } \\
\text { posttest. }\end{array}$ & $\begin{array}{l}\text { Hepatitis C } \\
\text { prevention }\end{array}$ & $\begin{array}{l}\text { Peer } \\
\text { education }\end{array}$ & $\begin{array}{l}\text { Massachusetts county jail . } 25 \text { men } \\
\text { who spoke and wrote in English. }\end{array}$ & $\begin{array}{l}\text { Changes in self-reported } \\
\text { behaviour, knowledge, } \\
\text { relationship with teacher. }\end{array}$ & Not reported & $3 c$ \\
\hline
\end{tabular}

NOTE: Validity score: 1 = good internal validity, 2 = moderate internal validityand 3 = poor internal validity; $\mathrm{a}=$ highly relevant, $\mathrm{b}=$ of some relevance, and $\mathrm{c}=$ not very relevant. 
Table 2 Number of included studies by Country

\begin{tabular}{ll}
\hline Country & Number of studies \\
\hline USA & 20 \\
UK & 20 \\
Canada & 9 \\
Australia & 2 \\
Ireland & 1 \\
Israel & 1 \\
Moldova & 1 \\
Russia & 1 \\
Mozambique & 1 \\
South Africa & 1 \\
\hline
\end{tabular}

\section{Listeners}

Strong qualitative evidence supported individual health gains for those trained as Listeners or befrienders. Trained individuals reported that they were 'giving something back', doing something constructive with their time in prison and being of benefit to the system; this consequently had an effect on individuals' self-esteem, self-worth and confidence [19,23,32,36,56,61]. Moreover, enhanced skills as a result of being a peer deliverer, like listening and communication, were mentioned by two studies $[56,61]$ and there were indications of prisoners feeling able to put these skills into practice on release from the institution [61].

There were some negative health effects reported $[32,56,61,92]$ and these related to the emotional burden of listening to other prisoners' problems and issues.

Two interventions studied in the UK, health trainers and peer mentors, focused on changing behaviours. One study provided weak evidence that mentoring had positive effects on health behaviours, treatment adherence,

Table 3 Number of included studies by intervention mode

\begin{tabular}{ll}
\hline Intervention mode & Number of studies \\
\hline Peer education & 21 \\
Peer support & 14 \\
Listeners & 6 \\
Peer mentoring & 4 \\
Prison hospice volunteers & 3 \\
Peer advisors & 2 \\
Health trainers & 2 \\
Peer counselling & 2 \\
Peer outreach & 1 \\
Peer observers & 1 \\
Peer training & 1 \\
\hline
\end{tabular}

Table 4 Number of included studies by health topic

\begin{tabular}{ll}
\hline Health topic & Number of studies \\
\hline HIV/AIDS/HCV/BBV prevention & 20 \\
General health, hygiene & 12 \\
Emotional support & 8 \\
Suicide/self harm prevention & 7 \\
Employment/housing post release & 4 \\
Mental health/substance abuse & 2 \\
Improving educational skills & 2 \\
Parenting & 1 \\
Violence reduction & 1 \\
\hline
\end{tabular}

drug taking and re-offending [83]. Two studies provided moderate evidence that becoming a health trainer positively affected knowledge, attitudinal and behaviour change, self-esteem and development of transferable skills [54,70]. There was little evidence of effects on health trainers' clients; however limited evidence showed that health trainers discussed a range of lifestyle issues with clients and referred them to other services [54,70].

Twenty-one predominantly qualitative studies $[19,23$, $29,32,36,53-59,61,64,68,72,79-81,96,102]$ indicated that being a peer worker was associated with positive effects on mental health and its determinants. These findings were consistent across a number of different models including peer education, peer support, Listeners, prison hospice volunteers, health trainers, and Peer Advisors. Skill development, including transferable employment skills, was also mentioned in relation to Peer Advisors [57,59] and health trainers [54]. Negative effects for peer workers related to experiencing a burden of care, particularly in roles involving emotional support $[32,56,61,92]$.

\section{Review Question 3: What is the effectiveness of peer delivery compared to professional delivery?}

Very few studies compared peer-led to professionally-led interventions. Three of four quantitative studies were about peer education for HIV prevention $[21,63,84]$, two of which were RCTs $[63,84]$. Consistent evidence from these studies indicated that peer educators are as effective as professional educators in HIV prevention. The fourth was a study of peer observers for suicide watch [86].

Consistent evidence from ten qualitative studies [23,54, $56-59,67,80,95,96]$ indicated that peer delivery was preferred to professional, with cross cutting themes including peer deliverers demonstrating empathy due to lived experiences, being non-judgemental, being trusted by prisoners and offering more time than staff. Prisoners felt more at ease talking to fellow prisoners and also found them more accessible. 
Table 5 Types of peer interventions

\begin{tabular}{|c|c|}
\hline $\begin{array}{l}\text { Type of peer } \\
\text { intervention }\end{array}$ & Working definition \\
\hline Peer education & $\begin{array}{l}\text { Peer education involves the teaching and communication of health information, values and behaviours between individuals } \\
\text { who are of equal social status, or share similar characteristics, or have common experiences }[103,104] \text {. Peer education has } \\
\text { been widely applied in the prison setting, particularly in relation to HIV prevention and risk reduction. Peer educators } \\
\text { typically undertake formal training to equip them with the knowledge and skills to undertake the role. }\end{array}$ \\
\hline Peer support & $\begin{array}{l}\text { Peer support is the support provided and received by those who share similar attributes or types of experience. Peer } \\
\text { support can be an informal process between individuals and/or can be provided through formalised interventions where } \\
\text { peer supporters seek to promote health and/or build people's resilience to different stressors [104]. There is a range of } \\
\text { different peer support interventions reported in the prison literature. In the UK, the Listeners scheme is a specific peer } \\
\text { support intervention focused on prevention of suicide and self-harm. }\end{array}$ \\
\hline $\begin{array}{l}\text { Prison hospice } \\
\text { volunteers }\end{array}$ & $\begin{array}{l}\text { Prison hospice volunteers provide companionship, practical assistance and social support to terminally ill patients. They may } \\
\text { be involved in a range of activities as requested by patients including letter writing, reading, accompanying patients to } \\
\text { religious services and other parts of prison and sometimes maintain a bedside vigil with dying patients [102]. }\end{array}$ \\
\hline Mentoring & $\begin{array}{l}\text { Mentoring describes the development of a relationship between two individuals where the mentee is able to learn from } \\
\text { the mentor, model positive behaviour and gain experience, knowledge or skills }[105,106] \text {. Peer mentors, as defined by } \\
\text { Finnegan et al., have a similar background or experiences to their mentee ([106]:6). There are a number of peer mentoring } \\
\text { schemes in UK prisons focused on education and training, such as The Learning Ladder [74], and on resettlement and } \\
\text { prevention of reoffending. }\end{array}$ \\
\hline Health trainers & $\begin{array}{l}\text { Health trainers are lay public health workers who use a client-centred approach to support individuals around health behav- } \\
\text { iour change and/or to signpost them to other services, some of which are also free at the point of delivery (Health Trainers } \\
\text { England). Prison health trainers receive the standardised training on health promotion, healthy lifestyles and mental health, } \\
\text { but adapted for the prison setting and client group. }\end{array}$ \\
\hline
\end{tabular}

\section{Review Question 4: What is the cost-effectiveness of peer based interventions in prisons?}

Only one study met the inclusion criteria [99,100], focusing on costs rather than health outcomes, and the programme aim was poorly described. The study showed management cost savings in prisons in the short term through the use of a Therapeutic Community (TC) programme, albeit these were a small part of the overall costs. Their findings suggest that TC activities or the existence of the TC environment may help to reduce or control prison management costs.

\section{Discussion}

Overall, current evidence is strongest in terms of evaluating effects on peer deliverers, rather than recipients of peer interventions. There is strong evidence that being a peer worker is associated with positive effects on mental health and its determinants, and this is consistent across a number of peer intervention models. Peer education interventions are effective in reducing risky behaviours, and peer support services are acceptable to prisoners and have a positive effect on recipients. There is some evidence that peer educators are as effective as professional educators for HIV prevention outcomes, and strong evidence that peer delivery is preferred to professional delivery. The finding of reduced risk of HIV transmission was in part reflective of the fact that it was the outcome that was most commonly evaluated. Therefore the absence of evidence for other health outcomes should not be misinterpreted as evidence of absence of the effectiveness of peer education for addressing health conditions other than HIV. Research into cost- effectiveness is sparse, with little economic evaluation even into interventions with evidence of effectiveness.

The 58 included studies represent the best available evidence, although their methodological quality was limited. Most did not report an underpinning theoretical model and only two defined 'peer', which leads the reader to make their own assumptions about whether peer deliverers and recipients within prisons are a homogeneous group. The dominance of positive findings in the quantitative data suggests publication bias. Clinical heterogeneity in outcomes and interventions precluded meta-analysis of most outcomes. Studies of interventions delivered by non-professionals, and studies of prison health, are not well indexed in electronic databases and early pilot searches returned impractically large numbers of hits. A more specific search strategy was developed, but this may have lost some sensitivity and therefore some relevant studies may have been missed. The effects of peer interventions on reoffending and other non-health outcomes (such as employment) are not represented in this review, nor are the effects of non-prisoner volunteers on prisoner health, effects of peer interventions in the probation service, or staff-tostaff peer interventions, although there is a body of literature on each of these. 63 studies were unobtainable: 17 were books and another substantial proportion were $\mathrm{PhD}$ theses or newspaper or magazine articles. Not all would have met inclusion criteria.

A previous review in this area highlighted a lack of evidence-based literature on the efficacy of prisonbased peer-led programmes [22], but this review only searched one database, and included only peer 
Table 6 Review Question 1 findings

\begin{tabular}{|c|c|c|c|c|c|c|}
\hline & \multicolumn{6}{|l|}{ Intervention type: } \\
\hline & Peer Education & Peer support & Listeners & $\begin{array}{l}\text { Prison hospice } \\
\text { volunteers }\end{array}$ & Peer mentoring & Health trainers \\
\hline \multirow[t]{5}{*}{ Knowledge } & $\begin{array}{l}\text { Ten studies } \\
{[66,68,69,84,88,90,93,97,98]}\end{array}$ & \multirow{5}{*}{$\begin{array}{l}\text { Two qualitative } \\
\text { studies showed } \\
\text { reported increases in } \\
\text { knowledge [58,80]. In } \\
\text { one of these studies, } \\
\text { a number of } \\
\text { respondents noted } \\
\text { that knowledge } \\
\text { acquired from the } \\
\text { training was } \\
\text { applicable to } \\
\text { improving } \\
\text { relationships with } \\
\text { their children, } \\
\text { partners and others } \\
\text { in the community } \\
\text { [58]. }\end{array}$} & \multirow{3}{*}{$\begin{array}{l}\text { Enhanced skills as a } \\
\text { result of being a } \\
\text { peer deliverer, like } \\
\text { listening and } \\
\text { communication, was } \\
\text { mentioned by two } \\
\text { studies [56,61] and } \\
\text { there was indication } \\
\text { of prisoners feeling } \\
\text { able to put these } \\
\text { skills into practice on } \\
\text { release from the } \\
\text { institution [61]. }\end{array}$} & & & $\begin{array}{l}\text { Two qualitative studies showed } \\
\text { increased knowledge on a } \\
\text { variety of topics, including: drugs, } \\
\text { sexual health, nutrition, alcohol } \\
\text { and mental health issues [54]. }\end{array}$ \\
\hline & $\begin{array}{l}\text { Statistically significantly higher } \\
\text { proportion of correct answers to } \\
22 / 43 \text { questions asked in peer } \\
\text { education vs control group. RR } \\
0.43 \text { ( } 95 \% \text { Cl: } 0.33,0.56,1 \text { study } n \\
=949) \text { to } 3.06 \text { (95\% Cl: } 1.91,4.91 \text {, } \\
1 \text { study, } n=200) .\end{array}$ & & & & & $\begin{array}{l}\text { Improvements were seen in the } \\
\text { mean knowledge scores in all } \\
\text { areas in one study [54], but it } \\
\text { was not possible to ascertain } \\
\text { whether these improvements } \\
\text { were statistically significant. }\end{array}$ \\
\hline & $\begin{array}{l}\text { Knowledge scores: mean } \\
\text { difference } 0.46(95 \% \mathrm{Cl}: 0.36,0.56 \text {, } \\
\left.2 \text { studies, } n=2494, \mathrm{I}^{2}=94 \%\right)\end{array}$ & & & & & $\begin{array}{l}\text { Both health trainers and Health } \\
\text { Trainer tutors reported that } \\
\text { Health Trainers had developed } \\
\text { effective communication and } \\
\text { listening skills as well as fostering } \\
\text { attributes essential for team } \\
\text { working and future employment } \\
\text { after release from prison [54]. }\end{array}$ \\
\hline & $\begin{array}{l}\text { Other evidence: peer educators } \\
\text { improved their own knowledge } \\
{[55,68,76] \text {. and [69] information }} \\
\text { was diffused to those outside the } \\
\text { prison, such as family members } \\
\text { and children. }\end{array}$ & & & & & \\
\hline & $\begin{array}{l}\text { In the study on literacy [88], > } \\
90 \% \text { of learners agreed that their } \\
\text { reading and communication skills } \\
\text { had improved. }\end{array}$ & & & & & \\
\hline \multirow[t]{2}{*}{ Intentions } & Four studies $[66,69,84,93]$ & & $\begin{array}{l}\text { In one study [37] } \\
61 \% \text { of those } \\
\text { surveyed said they } \\
\text { could talk to a } \\
\text { Listener about } \\
\text { anything that was } \\
\text { worrying them. } 74 \% \\
\text { had no problems } \\
\text { contacting a Listener } \\
\text { when they had } \\
\text { requested help. }\end{array}$ & & & \\
\hline & $\begin{array}{l}\text { One RCT [84] reported } \\
\text { improvements in: interest in }\end{array}$ & & $\begin{array}{l}57 \% \text { of users thought } \\
\text { they would seek the }\end{array}$ & & & \\
\hline
\end{tabular}

idence: peer educators mproved their own knowledge [5,68,76]. and [69] information was diffused to those outside and children

In the study on literacy [88]

$\%$ of learners agreed that their reading and communication skills 
Table 6 Review Question 1 findings (Continued)

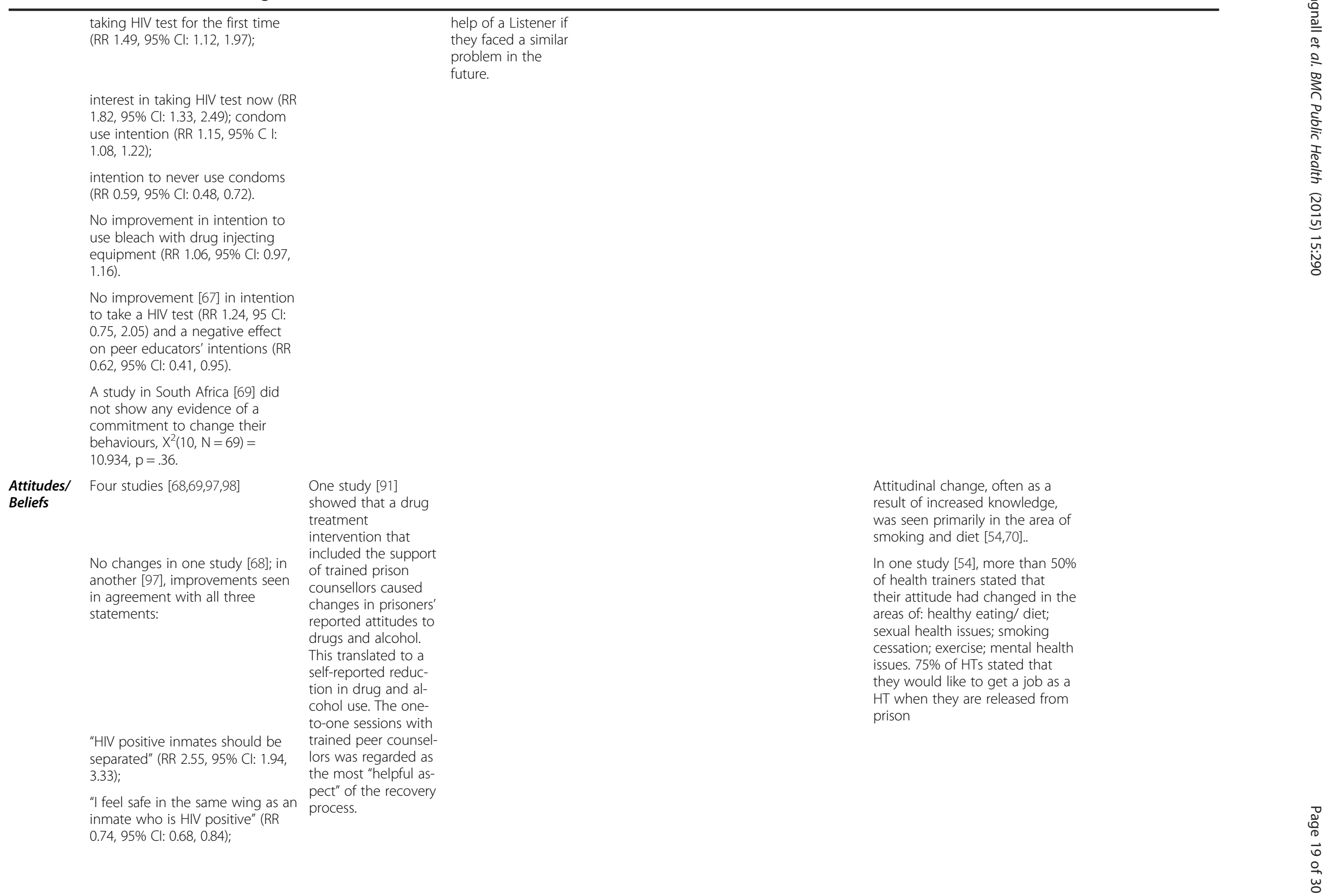


Table 6 Review Question 1 findings (Continued)

I know enough to protect myself from catching HIV/AIDS" (RR 0.54,

$95 \%$ C: $0.50,0.59)$

Behaviour Eleven studies

$[21,25,27,60,63,66,68,69,87,89,90,98]$

Positive effects seen:

Not using a condom at firs intercourse after release from prison (RR $0.73,95 \%$ Cl: $0.61,0.88$ 2 studies, $\mathrm{n}=400)$

injecting drugs after release from prison (RR 0.66, 95\% Cl: 0.53, 0.82 2 studies, $\mathrm{n}=400$ );

injected in past 4 weeks (RR 0.11 $95 \%$ Cl: $0.01,0.85,1$ study,

$\mathrm{n}=241)$;

sharing injection equipment afte release from prison (RR 0.33, 95\% Cl: $0.20,0.54,2$ studies, $n=400$ ):
In one study [92],

$64 \%$ of 22 prisoners

claimed that friends

and family had

noticed a difference

in their demeanou

inding them more

relaxed, responsible,

speak more and

more able to listen.

$73 \%$ agreed that

their new

responsibilities would

llow them to 'adjust

better' on release,

and $55 \%$ agreed that

the 'prison

authorities'

appreciated their

work. $77 \%$ said there

was a difference in

how immediate staff

interacted with

them: being trusted

more, staff talking

more to them, staff

being grateful for the

work they do. 86\%

said that fellow

prisoners behaved

differently towards

them.
In one study [83] At

3 months, 38/44

participants (86\%)

were receiving

outpatient psychiatric

services and 40/4

(91\%) successfully

managing the

medications.

At 6 months, 36/44

participants (82\%)

were medication

compliant, and 35/44

(80\%) demonstrated

symptom reduction.

$12 / 44(27 \%)$ had not

$12 / 44$ (27\%) had not

at 6 month time

point. 17/22 (77\%)

participants released

for at least

12 months had not

been rearrested. 16/

22 participants 4

had been released

for at least

12 months (73\%)

were abstinent in use

of alcohol or illegal

drugs or misuse of

prescription drugs.
Health trainers reported eating Peer training: One more fruit and vegetables and one health trainer had given up smoking $[54,70]$

Pudy [71] reported a statistically

significantly reduced rate of confrontation post-intervention at 0.432 (Cl: 0.319, 0.583, $\mathrm{p}<0.0005$ ). 
peer educators never having had

an HIV test (RR 0.31, 95\% Cl: 0.12

$0.78,1$ study, $n=847$ )

In one Russian study [27] the

prevalence of tattooing in prison

significantly decreased (42\% vs

$19 \%, p=0.03$ ) and of those who

were tattooed the proportion

using a new needle increased

from $23 \%$ to $50 \%$.

Where behaviour was measured

on a scale $[60,69,98]$, positive

effects were seen in all thre

studies.

HIV tests in prison [87] was

associated with having attended a HIV prevention programme in prison $(\mathrm{OR}=2.81,95 \% \mathrm{Cl}: 1.09$,

7.24).

Chlamydia screening in the

under-25 s rose from 13 to 83 in

a 6 month period after beginning

a peer education intervention,

similarly hepatitis $C$ screening in-

creased from 9 to 46 , and num-

bers were also increased for HIV

screening and hepatitis $B$ vaccinations [89].

In a study on parenting skills [90]

statistically significant

improvements in self-reported

father/ child contact were see

(mean difference $41.3,95 \% \mathrm{Cl}$ : $6.47,76.13)$.

Confidence One study [69] reported no significant differences.

No statistically

significant effect of Trained individuals

in three studies

reported that they

were 'giving

$[58,80,95]$ (WMD 1.51, doing something

95\% Cl: $-0.84,3.86,3$ constructive with

studies, $\mathrm{n}=83, \mathrm{I}^{2}=$

their time in prison

and being of benefit

to the system; this

consequently had an

effect on individuals'

self-esteem, self-

worth and
Volunteers

experience increases

in self-esteem and

self-worth as a result

of the service they

provide to others

[72,102]. Evidence

also suggests pris-

oners gain an en-

hanced sense of

compassion for

other people

$[72,102]$ and being
Health trainers seemed most

confident in signposting to

exercise, smoking cessation and

drugs services and least

confident in signposting to self-

harm, immunisation and dental

services [54].
Peer training: One study [71] reported s mall but statistically significant negative effects of the intervention on selfesteem (MD -2.15, 95\% Cl: $-4.20,-0.10)$, measured with the Rosenberg self-

esteem scale, and
optimism (MD 1.30, $95 \%$ Cl: $-0.83,3.43)$ 
Table 6 Review Question 1 findings (Continued)

\begin{tabular}{|c|c|c|c|c|}
\hline & $\begin{array}{l}\text { confidence } \\
{[19,23,32,36,56,61] .}\end{array}$ & $\begin{array}{l}\text { prison hospice } \\
\text { volunteers allows }\end{array}$ & & $\begin{array}{l}\text { measured with the } \\
\text { life orientation text. }\end{array}$ \\
\hline $\begin{array}{l}\text { Qualitative evidence } \\
\text { suggested } \\
\text { improvements in the } \\
\text { peer deliverers' self- } \\
\text { esteem, self-worth } \\
\text { and confidence as a } \\
\text { result of the role } \\
{[53,58,79-81,96] \text {.The }} \\
\text { sense of being } \\
\text { trusted by the prison }\end{array}$ & & $\begin{array}{l}\text { individuals 'to give } \\
\text { something } \\
\text { back' [77]. }\end{array}$ & $\begin{array}{l}\text { Qualitative research [54] found } \\
\text { that training as a health trainer } \\
\text { had been a huge boost to } \\
\text { prisoners' confidence, self-esteem } \\
\text { and self-worth, reported by key } \\
\text { staff. There was also evidence of } \\
\text { health trainers bolstering other } \\
\text { prisoners' reported self-esteem } \\
\text { and confidence through listening } \\
\text { and supporting individuals [54]. }\end{array}$ & $\begin{array}{l}\text { Peer outreach: } \\
\text { Qualitative evidence } \\
\text { suggested that peer } \\
\text { volunteers felt that } \\
\text { their role was } \\
\text { worthwhile and that } \\
\text { they were making a } \\
\text { difference to the } \\
\text { health of the prison } \\
\text { population [85]. }\end{array}$ \\
\hline
\end{tabular}

Peer advisers: Two

studies reported

increased self-esteem and self-confidence, coupled with peer deliverers reporting that they were building a work ethic and a sense of control over their lives

[57,59]. The role was perceived by the volunteers to be worthwhile and purposeful as well as enabling social interaction with others and offering 'structure' to the prison day [57]

$\begin{array}{ll}\begin{array}{l}\text { Mental } \\ \text { health }\end{array} & \begin{array}{l}\text { No effect on anger or frustration } \\ \text { in the parenting skills study [92], } \\ \text { either immediately post- } \\ \text { intervention (MD 0.20, } 95 \% \mathrm{Cl} \text { : }\end{array}\end{array}$


Table 6 Review Question 1 findings (Continued)

\begin{tabular}{|c|c|c|c|c|}
\hline $\begin{array}{l}-1.42,1.82) \text { or at longer follow-up } \\
\text { (MD 1.40,-0.03, 2.83). }\end{array}$ & $\begin{array}{l}\text { [58] and in one study } \\
\text { it was demonstrated } \\
\text { that this type of } \\
\text { intervention could } \\
\text { be particularly } \\
\text { beneficial for } \\
\text { prisoners during the } \\
\text { early part of their } \\
\text { sentence [62]. Those } \\
\text { who had used peer } \\
\text { support reported } \\
\text { using it as an avenue } \\
\text { to vent and to } \\
\text { overcome feelings of } \\
\text { anxiety, loneliness, } \\
\text { depression and self- } \\
\text { injury [58,79,96] and } \\
\text { there were indica- } \\
\text { tions that this may } \\
\text { be potentially benefi- } \\
\text { cial in preventing sui- } \\
\text { cides in prison [53] }\end{array}$ & $\begin{array}{l}\text { distressed prisoners } \\
\text { and improving their } \\
\text { mental state. There is } \\
\text { anecdotal evidence } \\
\text { that suicide and self- } \\
\text { harm is reduced as a } \\
\text { result of the support } \\
\text { offered by peers act- } \\
\text { ing in this role. A } \\
\text { fourth study [37] } \\
\text { found } 44 \% \text { of users } \\
\text { of the Listener } \\
\text { scheme reported } \\
\text { that they always felt } \\
\text { better after confiding } \\
\text { in a Listener, while } \\
52 \% \text { felt better at } \\
\text { least 'sometimes'. } \\
84 \% \text { said they had al- } \\
\text { ways found the ex- } \\
\text { perience helpful. }\end{array}$ & $\begin{array}{l}\text { own mortality as a } \\
\text { result of their } \\
\text { involvement [64]. } \\
\text { Moreover, the } \\
\text { recipients of one of } \\
\text { the programmes } \\
\text { suggested how the } \\
\text { volunteers had } \\
\text { supported them and } \\
\text { enabled them to } \\
\text { overcome states of } \\
\text { depression [64]. }\end{array}$ & $\begin{array}{l}\text { anger (mean } \\
\text { difference }-4.01,95 \% \\
\text { Cl: }-9.40,1.38) \text {, } \\
\text { measured with the } \\
\text { anger expression } \\
\text { scale. }\end{array}$ \\
\hline & & $\begin{array}{l}\text { Four studies } \\
{[32,56,61,92] \text { related }} \\
\text { the emotional } \\
\text { burden of listening } \\
\text { to other prisoners' } \\
\text { problems and issues. } \\
\text { Discussions relating } \\
\text { to suicidal intentions } \\
\text { and other distressing } \\
\text { topics could be } \\
\text { particularly } \\
\text { burdensome for peer } \\
\text { deliverers to manage. } \\
\text { There were also } \\
\text { reports of peer } \\
\text { deliverers } \\
\text { experiencing } \\
\text { 'burnout' and mental } \\
\text { exhaustion as a } \\
\text { result of the } \\
\text { demands placed on } \\
\text { their time by other } \\
\text { prisoners [56,92] }\end{array}$ & & $\begin{array}{l}\text { Peer support and } \\
\text { counseling: One } \\
\text { study [29] looked at } \\
\text { the effects of peer } \\
\text { support (Narcotics } \\
\text { Anonymous } \\
\text { meetings) and } \\
\text { counselling (12 step } \\
\text { programme), } \\
\text { compared to peer } \\
\text { support alone (NA } \\
\text { meetings only) on } \\
\text { mental health, } \\
\text { namely coherence, } \\
\text { meaning in life, } \\
\text { anxiety, depression } \\
\text { and hostility. } \\
\text { Improvements with } \\
\text { the combined } \\
\text { interventions were } \\
\text { seen in all outcomes: } \\
\text { coherence (mean } \\
\text { difference -0.31, } 95 \% \\
\text { Cl: -0.48, -0.14), } \\
\text { meaning in life (MD } \\
\text {-0.42, } 95 \% \text { Cl: -0.65, } \\
-0.19 \text { ), anxiety (MD }\end{array}$ \\
\hline
\end{tabular}


Preference In an American HIV RCT [84], 68\% preferred to be taught by an inmate with HIV versus $11 \%$ who preferred a HIV/ AIDS educator.

Additional Qualitative evidence suggested themes that peer deliverers found the
experience personally rewarding experience personally rewarding,

No statistically significant effect was seen on prisoners' perceptions of the meaning and purpose $[55,68]$. In prison environment one study, this included improved in the pooled results listening and communication of 3 studies $[58,80,95]$ skills as a result of their participation [90] Other

[55research suggested that being a peer educator also enabled the difficulties of prison life to be offset through the supportive network of other trained peer educators.
One study [79] found that $81 \%$ of 35

respondents valued

the existence of the

Peer Support Team

Another study [81]

reported that inmates

were very satisfied

with the quality of the

by PST members.

Expectations of the

PST were also well
met.

Staff reported that

PST members were

effective in handling

crisis interventions,

providing services to

inmates and serving

as role models.

In one study [81] PST

members estimated

that they provided

support to others of

3-5 hours per week

on average.
Prisoner outcomes: Issues most Peer observers: One

16/22 (73\%)

participants released

for at least

12 months were

employed, enrolled

in an educational

program or had

completed the

application process

for disability benefits.

18/22 (82\%)

likely to be discussed with hea

trainers were reported in one

controlled study [86] found a statistically

$(t(71.55)=2.14, p=$

$0.036)$ in the mean

number of hours on

watch following the

implementation of

the Inmate Observer

Onward referrals: Health

Programme.

participants who had trainers in one study [54] were

been released for at most likely to refer clients to

least 12 months had gym staff or healthcare staff.

secured treatment, Referrals were also made to

transitional housing Counselling, Assessment, Referral,

or a permanent Advice, and Throughcare services

place to live.

(CARATS), counsellor, dentist and

optician. 
Table 6 Review Question 1 findings (Continued)

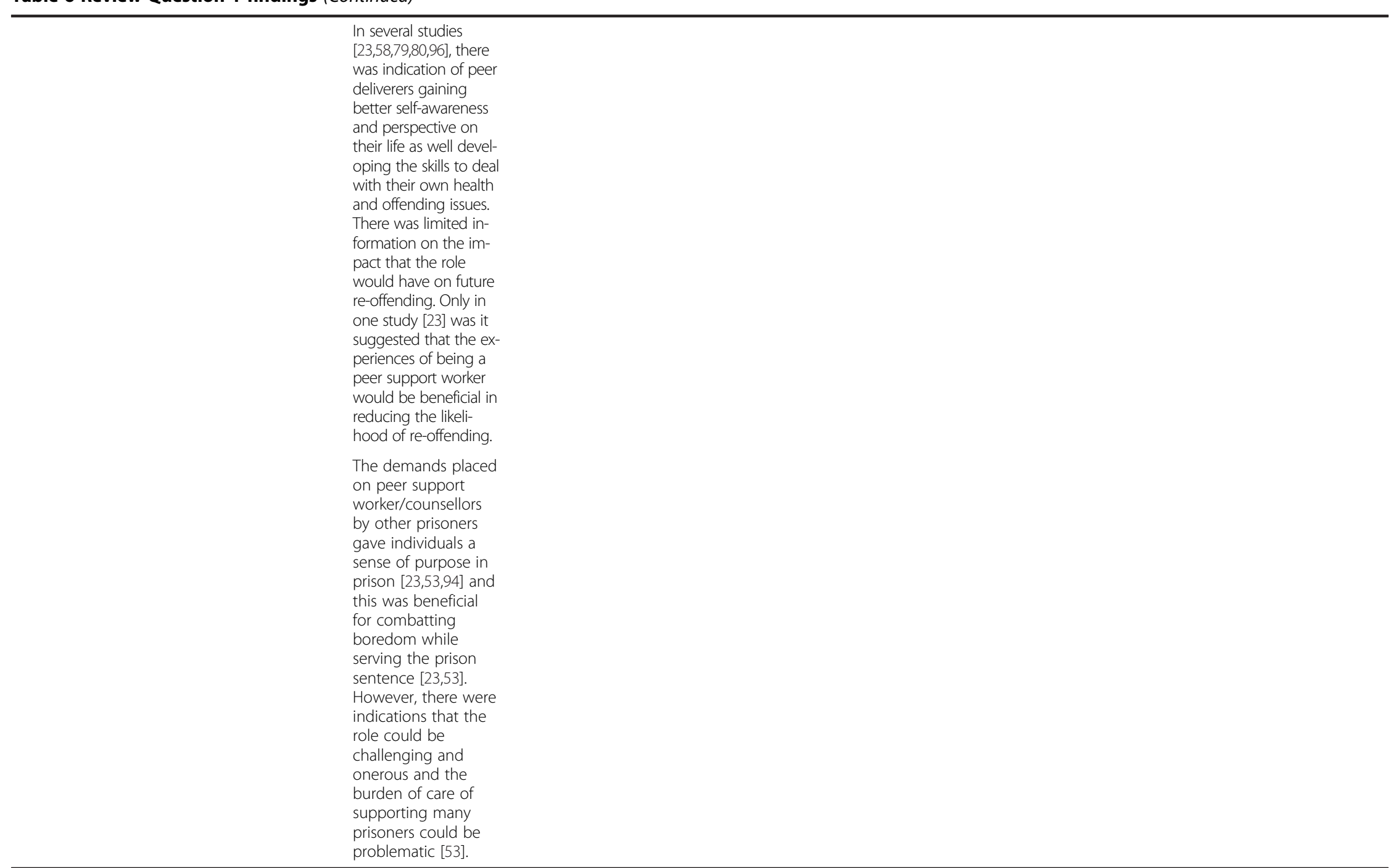




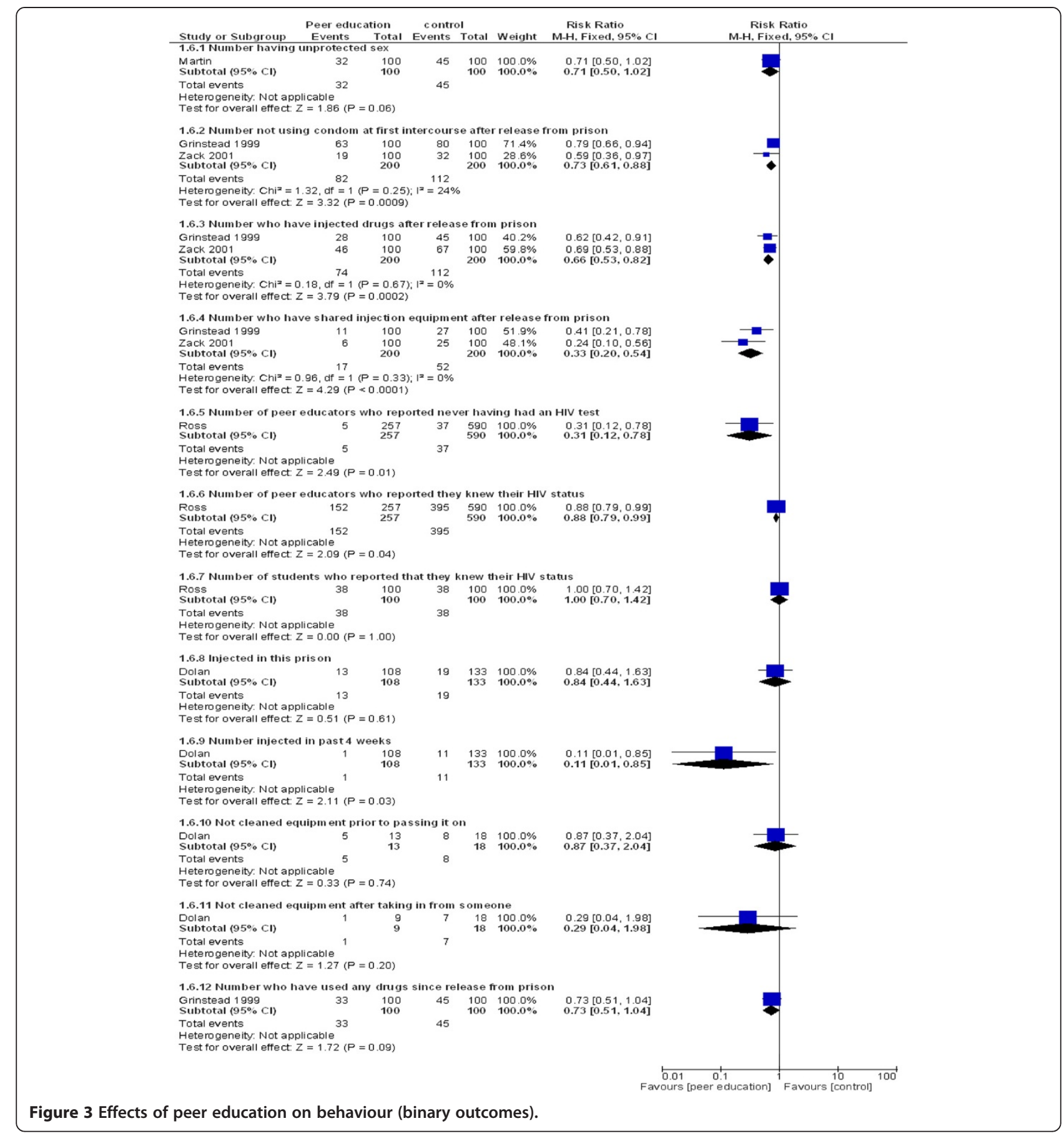

education interventions. Nevertheless, their conclusions concurred with ours, showing prison-based peer education programmes as well tolerated, effective and possibly more cost-effective than professionally led programmes. A 2011 systematic review of peer education for health promotion in prisons [31] searched fewer databases than our review, including only ten studies, and concluded, as does our review, that peer education is effective in reducing risk of HIV transmission.
This is the first systematic review of all the evidence on effectiveness and cost-effectiveness of peer interventions in prisons, a topic that is now of considerable interest to the Department of Health for England and Wales and NHS England. Given that the WHO consensus statement on mental health promotion in prisons argues that activities should be available to help offenders make best use of their time inside, and that the Prison Reform Trust estimates that only $20 \%$ of prisoners will 
be employed whilst inside (in industrial workshops for example), there is a need to provide meaningful occupation for offenders. Being a peer worker could provide such meaningful occupation [108], moreover peer-based interventions can be considered a valuable mechanism to maintain or improve health and wellbeing in the prison setting. A recent study of peer based interventions in mental health services found that peer workers were able to engage people with services by building relationships based on shared lived experience [109]. The benefits of peer education and support, particularly in those pathways that are concerned with changing behaviour or requiring individual motivation to pursue a healthy lifestyle, have also been seen in other areas such as managing substance misuse and addiction [110,111], and managing long-term conditions (for example, the Expert Patient Programme [112]).

This study has highlighted research gaps and ways in which the evidence base for peer-based interventions in prison settings could be strengthened. This work supports the Health and Justice function in Public Health England who have called for evidence-based guidelines and advice on all aspects of public health in prisons, including health promotion and public health [113]. It is vital that to further inform the evidence base, future studies need to be methodologically robust, sufficiently broad to capture outcomes for different stakeholder groups and assess costs and benefits both within and outside the prison system. Research is needed to explore the impact across the criminal justice system in line with the Department of Health's focus on offender health and understandings of the wider determinants of health in this vulnerable group.

There is also a pressing need for implementation and economic evaluation of a prison based peer educator initiative.

\section{Conclusions}

Peer-based interventions can be considered a valuable mechanism to maintain or improve health and wellbeing in the prison setting, with positive effects seen on knowledge and behaviour of peer deliverers and recipients. Peer education is less used in prisons in England and Wales than in the USA, perhaps reflecting more general trends in health promotion; however, the finding that peer education can increase knowledge and reduce risky health behaviours, particularly in relation to HIV prevention, suggests that consideration should be given to whether a peer education component should be introduced into other health behaviour change interventions.

\section{Transparency statement}

All authors had full access to all of the data and can take responsibility for the integrity of the data and the accuracy of the data analysis. Dr Bagnall affirms that the manuscript is an honest, accurate, and transparent account of the study being reported; that no important aspects of the study have been omitted; and that any discrepancies from the study as planned have been explained.

\section{Ethics approval statement}

The study received approval from the National Offender Management Service (NOMS) National Research Committee (Ref: 165-11) and the research team agreed to conduct the study in compliance with the Terms and Conditions set out by the National Research Committee. The study did not require ethical approval through NRES. Study documentation was reviewed through the Faculty of Health and Social Sciences Research Ethics Committee, Leeds Beckett University.

\section{Data sharing}

Full search strategies and lists of included and excluded studies are available from the corresponding author at a. bagnall@leedsbeckett.ac.uk.

\section{Abbreviations}

BBV: Blood Borne Viruses; HCV: Hepatits C Virus; Cl: Confidence Interval; DARE: Database of Abstracts of Reviews of Effects; DoPHER: Database of Promoting Health Evidence Reviews; EPPI: Evidence for Policy and Practice Information; HIV: Human Immunodeficiency Virus; HMP: Her Majesty's Prison; HSDR: Health Services and Delivery Research; NHS: National Health Service; NHSEED: National Health Service Economic Evaluation Database; NIHR: National Institute for Health Research; NOMS: National Offender Management Service; OHRN: Offender Health Research Network; PCT: Primary Care Trust; POA: Prison Officers Association; PORSCH: Prison and Offender Research in Social Care and Health; RePEc: Research Papers in Economics; WHO: World Health Organisation; YOI: Young Offenders' Institution.

\section{Competing interests}

All authors have completed the Unified Competing Interest form at http://www. icmje.org/conflicts-of-interest/ (available on request form the corresponding author) and declare that (1) all authors have support from NIHR HSDR stream for the submitted work (2); No authors have relationships with companies that might have an interest in the submitted work in the previous 3 years (3); their spouses, partners, or children have no financial relationships that may be relevant to the submitted work; and (4) No authors have non-financial interests that may be relevant to the submitted work.

\section{Authors' contributions}

AMB was project lead for the systematic review of effectiveness, co-wrote the review protocol, contributed substantially to the design of the review, undertook study selection, data extraction and validity assessment, designed data extraction and validity assessment told, undertook the meta-analysis, synthesis of qualitative and quantitative data, and wrote the paper. She is a guarantor. JS was the Principal Investigator for the study, having overall responsibility for the full report, was instrumental in the design and securing funding, co-wrote the review protocol, contributed to study selection and data synthesis and co-wrote the paper. She is a guarantor. $\mathrm{CH}$ was project lead for the review of cost-effectiveness, co-wrote the review protocol and contributed substantially to the design, read and commented on drafts of the paper. JW contributed substantially to the study design and undertook study selection, data extraction, validity assessment, qualitative synthesis, wrote sections of the full report, read and commented on drafts of the paper. KV-C undertook study selection, data extraction, validity assessment, synthesis and wrote up the cost-effectiveness review, read and commented 
on drafts of the paper. KK contributed to the design of the study, undertook study selection, data extraction and validity assessment and read and commented on drafts of the paper. GR undertook study selection, data extraction and validity assessment, meta-analysis, and read and commented on drafts of the paper. RD, NW and LH were involved in the design of the study and read and commented on drafts of the paper. All authors read and approved the final manuscript.

\section{Acknowledgements}

The authors would like to thank Ben Mitchell and Judy Wright for developing and carrying out the literature searches, and members of the steering and advisory groups for all their input: Caroline Thompson, Kathy Doran, Lee Stephenson, Dr Nick De Viggiani, Bill Penson, Professor Mike Kelly, Professor James Thomas and Dr Gerry Richardson.

Peers in Prison Settings is an independent study that is funded by the National Institute for Health Research Health Services and Delivery Research (NIHR HS\&DR) programme: Project: 10/2002/13.

The views and opinions expressed therein are those of the authors and do not necessarily reflect those of the HS \& DR programme, NIHR, NHS or the Department of Health. The study received approval from the National Offender Management Service National Research Committee and commenced on 1st February 2012.

\section{Author details}

${ }^{1}$ Centre for Health Promotion Research, Leeds Beckett University, Leeds LS1 $3 \mathrm{HE}$, UK. ${ }^{2}$ Academic Unit of Health Economics, Leeds Institute of Health Sciences, University of Leeds, LEEDS LS2 9LJ, UK. ${ }^{3}$ Spectrum Community Health CIC, White Rose House, West Parade, Wakefield WF1 1LT, UK. ${ }^{4}$ Leeds Community Healthcare HMP Leeds Healthcare Department, 2 Gloucester Terrace, Armley, Leeds LS12 2TJ, UK.

\section{Received: 28 August 2014 Accepted: 25 February 2015}

\section{Published online: 25 March 2015}

\section{References}

1. Rennie C, Senior J, Shaw J. The future is offender health: evidencing mainstream health services throughout the offender pathway. Crim Behav Ment Health. 2009;19:1-8.

2. Senior J, Shaw J. Prison healthcare. In: Jewkes Y, editor. Handbook on prisons. Cullompton: Willan Publishing; 2007. p. 377-98.

3. Department of Health. Choosing health. Making healthier choices easier. London: The Stationary Office; 2004. Cm 6732.

4. Social Exclusion Unit. Reducing re-offending by ex-prisoners. London: Crown; 2002.

5. Ministry of Justice. Population and capacity briefing for 6th December, 2013. London: Ministry of Justice; 2013.

6. Walmsley R. World prison population list (9th edn). London: International Centre for Prison Studies; 2011.

7. Ginn S. Elderly prisoners. BMJ. 2012;345:e6263.

8. Walmsley R. World prison population list (10th edn). London: International Centre for Prison Studies; 2013.

9. Fazel S, Danesh J. Serious mental disorder in 23,000 prisoners: a systematic review of 62 surveys. Lancet. 2002;359(9306):545-50.

10. Watson R, Stimpson A, Hostick T. Prison health care: a review of the literature. Int J Nurs Stud. 2004;41(2):119-28.

11. Liebling A. Prison suicide and its prevention. In: Jewkes $Y$, editor. Handbook on prisons. Cullompton: Willan Publishing; 2007. p. 423-46.

12. MacAskill S, Hayton P. Stop smoking support in HM prisons: the impact of nicotine replacement therapy. Stirling: University of Stirling and Open University; 2007.

13. Stewart $D$. The problems and needs of newly sentenced prisoners: results from a national survey. London: Ministry of Justice; 2008.

14. Harris F, Hek G, Condon L. Health needs of prisoners in England and Wales: the implications for prison healthcare of gender, age and ethnicity. Health Soc Care Community. 2007:15:56-66.

15. England NHS. National Partnership Agreement Between: The National Offender Management Service, NHS England and Public Health England for the Co-Commissioning and Delivery of Healthcare Services in Prisons in England. London: NHS England; 2013.

16. Department of Health. Inclusion Health: Improving the way we meet the primary health care needs of the socially excluded. London: Department of Health; 2010.
17. Ginn S. Promoting health in prisons. BMJ. 2013;346:f2216.

18. Patel $\mathrm{L}$. The Patel report: Reducing drug-related crime and rehabilitating offenders. London: Prison Drug Treatment Strategy Review Group; 2010.

19. Levenson J, Farrant F. Unlocking potential: active citizenship and volunteering by prisoners. Probation J. 2002;49(3):195-204.

20. Snow $L$, Biggar $K$. The role of peer support in reducing self-harm in prisons. In: Dear GE, editor. Preventing suicide and other self-harm in prison. New York: Palgrave; 2006. p. 153-66.

21. Zack B, Bancroft C, Blea L, Comfort M, Grossman N, OG. Collaborative research to prevent HIV among male prison inmates and their female partners. Science to Community, Prevention no. 7, revised December 2004, p. 1-4.

22. Devilly GJ, Sorbello L, Eccleston L, Ward T. Prison-based peer-education schemes. Aggress Violent Behav. 2005;10(2):219-40.

23. Edgar K, Jacobson J, Biggar K. Time Well Spent: A practical guide to active citizenship and volunteering in prison. Prison Reform Trust: Prison Reform Trust London; 2011.

24. Daigle MS, Daniel AE, Dear GE, Frottier P, Hayes LM, Kerkhof A, et al. Preventing suicide in prisons, part II - International comparisons of suicide prevention services in correctional facilities. Crisis. 2007;28(3):122-30.

25. Grinstead OA, Zack B, Faigeles B, Grossman N, Blea L. Reducing postrelease HIV risk among male prison inmates - A peer-led intervention. Crim Justice Behav. 1999;26(4):453-65.

26. Sirdifield C, Bevan L, Calverley M, Mitchell L, Craven J, Brooker C. A quide to implementing the new futures health trainer role across the criminal justice system. University of Lincoln: Lincoln; 2007.

27. Dolan KA, Bijl M, White B. HIV education in a Siberian prison colony for drug dependent males. Int J Equity Health. 2004;3(7):3-7.

28. Vaz RG, Gloyd S, Trindade R. The effects of peer education on STD and AIDS knowledge among prisoners in Mozambique. Int J STD AIDS. 1996;7(1):51-4.

29. Chen G. Social support, spiritual program, and addiction recovery. Int J Offender Ther Comp Criminol. 2006;50(3):306-23.

30. Woodall J, Dixey R. Advancing the health promoting prison: a call for global action. Global Health Promotion (In Press). 2015;ISSN 1757-9759.

31. Wright N, Bleakley A, Butt C, Chadwick O, Mahmood K, Patel K, et al. Peer health promotion in prisons: a systematic review. Int J Prison Health. 2011;7(4):37-51.

32. Davies B. The Swansea Listener Scheme: views from the prison landings. Howard J Crim Justice. 1994:33(2):125-35.

33. South J, Raine G, White J. Community Health Champions: Evidence Review. Leeds: Centre for Health Promotion Research, Leeds Metropolitan University; 2010.

34. Lewin SA, Babigumira SM, Bosch-Capblanch X, Aja G, van Wyk B, Glenton C, et al. Lay health workers in primary and community care: a systematic review of trials. London: London School of Hygiene \& Tropical Medicine; 2006

35. Service NOM. Discussion Document. NOMS Commissioning Intentions 2012-13. Version 2. London: Ministry of Justice; 2012

36. Hall B, Gabor P. Peer suicide prevention in a prison. Crisis. 2004;25(1):19-26.

37. Snow $L$. The role of formalised peer group support in prisons. In: Towl G, Snow L, McHugh M, editors. Suicide in prisons. Oxford: Blackwell Publishers; 2002.

38. Emmons K, Puleo E, Park E, Gritz E, Butterfield R, Weeks J, et al. Peer-delivered smoking counseling for childhood cancer survivors increases rate of cessation: the partnership for health study. J Clin Oncol. 2005;23(27):6516-23.

39. Miller T, Zaloshnja E, Spicer R. Effectiveness and benefit-cost of peer-based workplace substance abuse prevention coupled with random testing. Accid Anal Prev. 2007;39(3):565-73.

40. Stant A, Castelein S, Bruggeman R, van Busschbach J, van der Gaag M, Knegtering $\mathrm{H}$, et al. Economic aspects of peer support groups for psychosis. Community Ment Health J. 2011:47(1):99-105.

41. South J, Bagnall A, Hulme C, Woodall J, Longo R, Dixey R, et al. A systematic review of the effectiveness and cost-effectiveness of peer-based interventions to maintain and improve offender health in prison settings. Health Serv Deliv Res. 2014;2(35).

42. Centre for Reviews Dissemination. Systematic Reviews. CRD's guidance for undertaking reviews in health care. York: University of York; 2009.

43. Higgins J, Green S. Cochrane Handbook for Systematic Review of Interventions version 5.1.0. The Cochrane Collaboration; 2011. Available from www.cochrane-handbook.org.

44. National Institute for Health and Clinical Excellence. Methods for the development of NICE public health guidance (second edition). London: National Institute for Health and Clinical Excellence; 2009. 
45. South J, Meah A, Bagnall A-M, Kinsella K, Branney P, White J, et al. People in Public Health - a study of approaches to develop and support people in public health roles. London: NIHR Service Delivery and Organisation Programme; 2010.

46. Thomas J, Harden A. Methods for the thematic synthesis of qualitative research in systematic reviews. BMC Med Res Methodol. 2008;8(45):1-10.

47. Seale C, Silverman D. Ensuring rigour in qualitative research. Eur J Public Health. 1997;7(4):379-84.

48. Thomas J, Harden A, Oakley A, Oliver S, Sutcliffe K, Rees R, et al. Integrating qualitative research with trials in systematic reviews: an example from public health. Br Med J. 2004;328:1010-2.

49. Rees R, Oliver K, Woodman J, Thomas J. Children's views about obesity, body size, shape and weight: a systematic review. London: EPPI Centre, Social Science Research Unit, Institute of Education, University of London; 2009.

50. Drummond M, Sculpher M, Torrance G, O'Brien B, Stoddart G. Methods for the economic evaluation of health care programmes. 3rd edition 2005.

51. Ramsey S, Willke R, Briggs A, Brown R, Buxton M, Chawla A, et al. Good research practices for cost-effectiveness analysis alongside clinical trials: the ISPOR RCT-CEA Task Force report. Value Health. 2005;8(5):521-33.

52. Ryan RE, Kaufman CA, Hill SJ. Building blocks for meta-synthesis: data integration tables for summarising, mapping, and synthesising evidence on interventions for communicating with health consumers. BMC Med Res Methodol. 2009;9(16) http://dx.doi.org/10.1186/1471-2288-9-16.

53. Boothby MRK. Insiders' Views of their Role: Toward their Training. Canadian J Criminol Crim Justice. 2011;53(4):424-48.

54. Brooker S. New futures Health trainers: an impact assessment. Lincoln: University of Lincoln; 2007.

55. Collica K. Surviving incarceration: Two prison-based peer programs build communities of support for female offenders. Deviant Behav. 2010;31(4):314-47.

56. Foster J. Peer support in prison health care. An investigation into the Listening scheme in one adult male prison. School of health \& social care, University of Greenwich, 2011 Sept-Dec 2011. Report No.

57. Hunter G, Boyce I. Preparing for employment: prisoners' experience of participating in a prison training programme. Howard J Crim Justice. 2009;48:117-31.

58. Blanchette K, Eljdupovic-Guzina G. Results of a Pilot Study of the Peer Support Program for Women Offenders. Canada: Correctional Service of Canada; 1998.

59. Boyce I, Hunter G, Hough M. Peer advice project. An evaluation. London: St Giles Trust, King's College; 2009.

60. Bryan A, Robbins RN, Ruiz MS, O'Neill D. Effectiveness of an HIV Prevention Intervention in Prison Among African Americans, Hispanics, and Caucasians. Health Educ Behav. 2006;33(2):154-77.

61. Dhaliwal R, Harrower J. Reducing prisoner vulnerability and providing a means of empowerment: evaluating the impact of a listener scheme on the listeners. Br J Forensic Pract. 2009;11(3):35-43.

62. Jacobson J, Edgar K, Loucks N. There when you need them most: Pact's first night in custody services. London: Prison Reform Trust; 2008.

63. Martin SS, O'Connell DJ, Inciardi JA, Surratt HL, Maiden KM. Integrating an HIV/HCV brief intervention in prisoner reentry: results of a multisite prospective study. J Psychoactive Drugs. 2008:40(4):427-36.

64. Maull FW. Hospice care for prisoners: establishing an inmate-staffed hospice program in a prison medical facility. Hospice J. 1991;7(3):43-55.

65. Munoz-Plaza CE, Strauss SM, Astone JM, Des Jarlais DC, Hagan H. Hepatitis C Service Delivery in Prisons: Peer Education From the "Guys in Blue.". J Correct Health Care. 2005;11(4):347-68.

66. Ross MW, Harzke AJ, Scott DP, McCann K, Kelley M. Outcomes of Project Wall Talk: an HIV/AIDS peer education program implemented within the Texas State Prison system. AIDS Educ Prev. 2006;18(6):504-17.

67. Schinkel W. Routes out of prison using life coaches to assist resettlement. Howard J Crim Justice. 2012;4(51):359-71.

68. Scott DP, Harzke AJ, Mizwa MB, Pugh M, Ross MW. Evaluation of an HIV Peer Education Program in Texas Prisons. J Correct Health Care. 2004;10(2):151-73.

69. Sifunda S, Reddy PS, Braithwaite R, Stephens T, Bhengu S, Ruiter RA, et al. The effectiveness of a peer-led HIV/AIDS and STI health education intervention for prison inmates in South Africa. Health Educ Behav. 2008;35(4):494-508.

70. Sirdifield C. Piloting a new role in mental health - prison based health trainers. J Mental Health Workforce Dev. 2006;1(4):15-22.
71. Walrath C. Evaluation of an Inmate-Run Alternatives to Violence Project: The Impact of Inmate-to Inmate Intervention. J Interpers Violence. 2001;16(7):697-711.

72. Wright KN, Bronstein L. Creating decent prisons: a serendipitous finding about prison hospice. J Offender Rehabil. 2007:44(4):1-16.

73. Mentor2work. Mentor2work. An action research project at HMP Liverpool into peer mentoring and employment for offenders with mental health problems. London: Centre for Mental Health; 2005 URL: www.scmh.org.uk/ pdfs/mentoring_liverpool_report.doc (accessed April 2014).

74. The Learning Ladder Ltd. Mentoring for Progression: Peer Mentoring in a YOI. HMYOI Reading; 2010. URL: http://www.ingentaconnect.com/content/ openu/jwpll/2010/00000012/00000003/art00006?token=004612c0754058474 47b49762f2a407276386b79214833757e6f3f2f2730673f582f6b (Accessed April 2015).

75. Ashton C. Healing from the inside: an analysis of correctional service of Canada's circle of knowledge Keepers Program. 2010.

76. Betts-Symond G. Community based health and first aid in Action in Irish Red Cross Prisoners. 3 Year Evaluation. Dublin: Irish Prison Service; 2011.

77. Cichowlas JA, Chen Y-J. Volunteer prisoners provide hospice to dying inmates. Ann Health Law. 2010;19(1 Spec No):127-32.

78. Collica K. The prevalence of HIV peer programming in American prisons: An opportunity wasted. J Correct Health Care. 2007;13(4):278-88.

79. Correctional Service of Canada. Opening the window on a very dark day: A program evaluation of the Peer Support Team in the Kingston Prison for women. 2009

80. Delveaux K, Blanchette K. Results of an Evaluation of the Peer Support Program at Nova Institution for Women. Ottawa, ON: Research Branch, Correctional Service of Canada; 2000. URL: http://www.csc-scc.gc.ca/ research/r87-eng.shtml.

81. Eamon KC, McLaren DL, Munchua MM, Tsutsumi LM. The Peer support program at Edmonton Institution for women. Forum. 2012;11(3):28-30.

82. Farrin J. Prisoner Peer Support Program. (PowerPoint presentation). Adelaide: South Australian Department for Correctinal Services; n.d. (No URL details are available at present, but source material is available from the corresponding author on request).

83. Goldstein EH, Warner-Robbins C, McClean C, Macatula L, Conklin R. A peer-driven mentoring case management community reentry model: an application for jails and prisons. Fam Community Health. 2009;32(4):309-13.

84. Grinstead O, Faigeles B, Zack B. The effectiveness of peer HIV education for male inmates entering state prison. J Health Educ. 1997;28(6):31-7.

85. Hoover J, Jurgens R. Harm reduction in prison: The Moldova Model. New York, NY: Open Society Institute; 2009.

86. Junker G, Beeler A, Bates J. Using Trained Inmate Observers for Suicide Watch in a Federal Correctional Setting: A Win-Win Solution. Psychol Serv. 2005;2(1):20-7.

87. MacGowan R, Eldridge G, Sosman JM, Khan R, Flanigan T, Zack B, et al. HIV Counseling and Testing of Young Men in Prison. J Correct Health Care. 2006;12(3):203-13.

88. O'Hagan S. West Midlands Reading Network Pilot 2008-2010. London: Shannon Trust; 2011.

89. Peek H. Healthcare Representatives. WHO Health in Prisons Project (WHO HIPP) Best Practice Awards. 2011 Application; 2011. URL: http://www.uclan. ac.uk/research/explore/projects/assets/who_hipp_practice_awards_2011 _england_cat2.pdf (accessed August 2012).

90. Penn State Erie. Final report of the process evaluation of the Long Distance Dads Program. 2001.

91. Player E, Martin C. ADT (Addictive Diseases Trust) Drug Treatment Programme at HMP Downview: A Preliminary Evaluation. London: Home Office; 1996.

92. Richman J. Listeners: inmate care workers and suicide policies in HM prisons. N2N: Nurse2Nurse. 2004;4(3):18-21.

93. Schlapman N, Cass PS. Project: HIV prevention for incarcerated youth in Indiana. J Community Health Nurs. 2000;17(3):151-8.

94. Stewart W. Evaluating peer social care training in prisons. Prison Serv J. 2011;195:43-6.

95. Syed, Blanchette. Results of an evaluation of the peer support program at Grand Valley Institution for women. Correctional Service of Canada, 2000.

96. Syed F, Blanchette K. Results of an evaluation of the peer support program at Joliette Institution for women. Correctional Service of Canada. 2000.

97. Taylor S. New South Wales Prison HIV Peer Education Program. Sydney: Department of Corrective Services Australia; 1994. 
98. Zucker D. Hepatitis C prevention in a county correctional facility. Gastroenterol Nurs. 2006;29(2):173.

99. Zhang S, Roberts REL, McCollister KE. An economic analysis of the in-prison therapeutic community model on prison management costs. J Crim Justice. 2009;37(4):388-95.

100. Huggins R. Mentoring for Progression: Prison Mentoring project - assessing strengths, outcomes and roll-out potential. 2010.

101. Sifunda S, Reddy PS, Braithwaite RB, Stephens T, Bhengu S, Ruiter RAC, et al. Social construction and cultural meanings of STI/HIV-related terminology among Nguni-speaking inmates and warders in four South African correctional facilities. Health Educ Res. 2006;22(6):805-14.

102. Wright KN, Bronstein L. Organizational Analysis of Prison Hospice. Prison J. 2007;87(4):391-407.

103. Milburn K. A critical review of peer education with young people with special reference to sexual health. Health Educ Res. 1995:10(4):407-20

104. Dennis C-L. Peer support within a healthcare context: a concept analysis. Int J Nurs Stud. 2003:40(3):321-32

105. Jolliffe D, Farrington DP. A Rapid Evidence Assessment of the Impact of Mentoring on Re-offending: A Summary. London: Home Office; 2007.

106. Finnegan L, Whitehurst D, Deaton S. Models of mentoring for inclusion and employment. Thematic review of existing evidence on mentoring and peer mentoring. London: Centre for Economic \& Social Inclusion; 2010.

107. Bowman VE, Lowrey L, Purser J. Two-Tiered Humanistic Pre-release Interventions for Prison Inmates. J Offender Rehabil. 1997;25(1/2):115-28.

108. Prison Reform Trust. Bromley briefings. Prison factfile. London: Prison Reform Trust; 2012

109. Gillard S, Edwards C, Gibson S, Holley J, Owen K. New ways of working in mental health services: a qualitative, comparative case study assessing and informing the emergence of new peer worker roles in mental health services in England. Health Serv Deliv Res. 2014;2(19).

110. Hunter G, Power R. Involving Big Issue vendors in a peer education initiative to reduce drug-related harm: a feasibility study. Drugs: Education, Prevention \& Policy. 2002;9(1):57-69.

111. Boisvert R, Martin L, Grosek M, Clarie A. Effectiveness of a peer-support community in addiction recovery: participation as intervention. Occup Ther Int. 2008;15(4):205-20.

112. Rogers A, Bower P, Gardner C, Kennedy A, Lee V, Middleton E, et al. The National Evaluation of the Pilot Phase of the Expert Patients Programme. Manchester: National Primary Care Research \& Development Centre; 2006.

113. National Offender Management Serivce, Public Health England, England N. National Partnership Agreement Between: The National Offender Management Service, NHS England and Public Health England for the CoCommissioning and Delivery of Healthcare Services in Prisons in England. NHS England Publications Gateway 2013 Contract No.: Reference 00435.

\section{Submit your next manuscript to BioMed Central and take full advantage of:}

- Convenient online submission

- Thorough peer review

- No space constraints or color figure charges

- Immediate publication on acceptance

- Inclusion in PubMed, CAS, Scopus and Google Scholar

- Research which is freely available for redistribution 